\title{
King-Type Derivative-Free Iterative Families: Real and Memory Dynamics
}

\author{
F. I. Chicharro, ${ }^{1}$ A. Cordero, ${ }^{1}$ J. R. Torregrosa, ${ }^{1}$ and M. P. Vassileva ${ }^{2}$ \\ ${ }^{1}$ Instituto Universitario de Matemática Multidisciplinar, Universitat Politècnica de València, València, Spain \\ ${ }^{2}$ Instituto Tecnológico de Santo Domingo (INTEC), Santo Domingo, Dominican Republic \\ Correspondence should be addressed to M. P. Vassileva; maria.penkova@intec.edu.do
}

Received 29 June 2017; Accepted 4 October 2017; Published 31 October 2017

Academic Editor: Guido Caldarelli

Copyright (c) 2017 F. I. Chicharro et al. This is an open access article distributed under the Creative Commons Attribution License, which permits unrestricted use, distribution, and reproduction in any medium, provided the original work is properly cited.

\begin{abstract}
A biparametric family of derivative-free optimal iterative methods of order four, for solving nonlinear equations, is presented. From the error equation of this class, different families of iterative schemes with memory can be designed increasing the order of convergence up to six. The real stability analysis of the biparametric family without memory is made on quadratic polynomials, finding areas in the parametric plane with good performance. Moreover, in order to study the real behavior of the parametric class with memory, we associate it with a discrete multidimensional dynamical system. By analyzing the fixed and critical points of its vectorial rational function, we can select those methods with best stability properties.
\end{abstract}

\section{Introduction}

The solution of a nonlinear equation $f(x)=0$ is a common problem in topics related to several disciplines of science. However, most of these equations can not be solved analytically. In this case, iterative methods acquire prominence, because they can reach an approximate solution of $f(x)$. Newton's scheme is the most-applied iterative method, but the evaluation of the derivative of $f$ is its most remarkable inconvenience.

Several authors have made great efforts to study the complex dynamics of some derivative-free iterative methods [1-3], but the analysis of the real dynamics is not as usual as the complex one $[4,5]$.

During the last years, many researchers have focused on the construction of optimal multipoint methods without memory based on the Kung and Traub's conjecture [6], which claims that to create an optimal method without memory with optimal order $2^{n}, n+1$ functional evaluations are required. Construction and development of multipoint methods with memory have more advantages in comparison with methods without memory. In other words, they are able to increase the convergence order without any new functional evaluation. Due to this basic fact, they can also get higher efficiency index (the efficiency index was defined by Ostrowski [7] as $I=p^{1 / d}$, where $p$ is the order of convergence and $d$ is the number of functional evaluations required per iteration). As another plus, they generate more stable computations than optimal methods without memory, even with higher convergence orders.

The basic idea for the construction of multipoint methods with memory was introduced by Traub [8], who presented a version with memory from Steffensen's method. Recently, based on this method, some schemes with memory have been developed by several authors. We can see interesting overviews in $[9,10]$.

In this paper, we deeply analyze the real dynamics of a version with memory of a King-type derivative-free iterative family. Two new tools are introduced in the real dynamics section: the unified parameter plane and the dynamical line. The former gathers the information of different parameters planes into one plane. The latter becomes a comfortable tool to visualize the dynamical behavior of a method for a set of initial points in the real line. 
Starting from King's family of fourth-order schemes

$$
\begin{aligned}
y_{k} & =x_{k}-\frac{f\left(x_{k}\right)}{f^{\prime}\left(x_{k}\right)}, \\
x_{k+1} & =y_{k}-\frac{f\left(x_{k}\right)+\alpha f\left(y_{k}\right)}{f\left(x_{k}\right)+(\alpha-2) f\left(y_{k}\right)} \frac{f\left(y_{k}\right)}{f^{\prime}\left(x_{k}\right)},
\end{aligned}
$$

where $\alpha$ is a disposable parameter, we design a derivative-free family of fourth-order methods. The order of convergence can be increased introducing memory, as can be deduced from the error equation.

In the manuscript, we use symbols $\sim$ and $O$ in the following way: if $\lim _{n \rightarrow \infty}\left(a_{n} / b_{n}\right)=C$, with $C$ being a nonzero constant, we write $a_{n}=O\left(b_{n}\right)$ or $a_{n} \sim b_{n}$. The notation and techniques used in the proofs of the results can be found in [9-11].

The rest of this paper is organized as follows. In Section 2, we introduce the derivative-free parametric family and its error equation. Section 3 addresses the increase of the order of convergence with the introduction of the memory in the iterative schemes. In Section 4, the real dynamics of the biparametric family is studied for different quadratic polynomials, while Section 5 covers the dynamical study of the methods with memory. Finally, Section 6 collects some conclusions about the obtained results.

\section{Parametric Families of Iterative Schemes}

Following the structure of King's family and replacing the derivative by a first-order divided difference we present the following schemes:

$$
\begin{aligned}
y_{k} & =x_{k}-\frac{f\left(x_{k}\right)}{f\left[x_{k}, w_{k}\right]}, \\
x_{k+1} & =y_{k}-\frac{f\left(x_{k}\right)+\alpha f\left(y_{k}\right)}{\mu f\left(x_{k}\right)+\beta f\left(y_{k}\right)} \frac{f\left(y_{k}\right)}{f\left[y_{k}, w_{k}\right]},
\end{aligned}
$$

where $w_{k}=x_{k}+\gamma f\left(x_{k}\right)$, with $\alpha, \beta, \gamma$, and $\mu$ being real parameters, $\gamma \neq 0$.

The order of convergence of methods (2) is established in the following result. The proof only requires the development in Taylor series of the elements of the iterative expression and some algebraic manipulations.

Theorem 1. Let us suppose that $f: I \subseteq \mathbb{R} \rightarrow \mathbb{R}$ is a sufficiently differentiable function in an open interval I and $x^{*} \in I$ is a simple root of $f(x)=0$. If the initial approximation $x_{0}$ is close enough to $x^{*}$, then the iterative scheme (2) has optimal fourthorder convergence when $\mu=1$ and $\beta=\alpha-1$ for all nonzero $\gamma$, being in this case the error equation

$$
\begin{aligned}
& e_{k+1} \\
& =\left(1+\gamma f^{\prime}\left(x^{*}\right)\right)^{2} c_{2}\left(2+\alpha+\alpha \gamma f^{\prime}\left(x^{*}\right) c_{2}^{2}-c_{3}\right) e_{k}^{4} \\
& \quad+O\left(e_{k}^{5}\right),
\end{aligned}
$$

where $e_{k}=x_{k}-x^{*}, k=0,1, \ldots$, and $c_{j}=(1 / j !)\left(f^{(k)}\left(x^{*}\right) /\right.$ $\left.f^{\prime}\left(x^{*}\right)\right), k \geq 2$.
Proof. By expanding in Taylor series $f\left(x_{k}\right)$, we get

$$
\begin{aligned}
& f\left(x_{k}\right)=f^{\prime}\left(x^{*}\right)\left[e_{k}+c_{2} e_{k}^{2}+c_{3} e_{k}^{3}+c_{4} e_{k}^{4}+c_{5} e_{k}^{5}+c_{6} e_{k}^{6}\right] \\
& +O\left(e_{k}^{7}\right) \\
& f\left[x_{k}, w_{k}\right]=f^{\prime}\left(x^{*}\right)\left[1+\left(2+\gamma f^{\prime}\left(x^{*}\right)\right) c_{2} e_{k}\right. \\
& +\left(\gamma f^{\prime}\left(x^{*}\right) c_{2}^{2}+\left(3+3 \gamma f^{\prime}\left(x^{*}\right)+\gamma^{2} f^{\prime}\left(x^{*}\right)^{2}\right) c_{3}\right) \\
& \cdot e_{k}^{2}+\left(2+\gamma f^{\prime}\left(x^{*}\right)\right)\left(2 \gamma f^{\prime}\left(x^{*}\right) c_{2} c_{3}+(2\right. \\
& \left.\left.+2 \gamma f^{\prime}\left(x^{*}\right)+\gamma^{2} f^{\prime}\left(x^{*}\right)^{2}\right) c_{4}\right) e_{k}^{3}+\left(\gamma^{2} f^{\prime}\left(x^{*}\right)^{2} c_{2}^{2} c_{3}\right. \\
& +\gamma f^{\prime}\left(x^{*}\right)\left(3+2 \gamma f^{\prime}\left(x^{*}\right)\right) c_{3}^{2}+\gamma f^{\prime}\left(x^{*}\right)(7 \\
& \left.+8 \gamma f^{\prime}\left(x^{*}\right)+3 \gamma^{2} f^{\prime}\left(x^{*}\right)^{2}\right) c_{2} c_{4}+\left(5+10 \gamma f^{\prime}\left(x^{*}\right)\right. \\
& \left.\left.+10 \gamma^{2} f^{\prime}\left(x^{*}\right)^{2}+5 \gamma^{3} f^{\prime}\left(x^{*}\right)^{3}+\gamma^{4} f^{\prime}\left(x^{*}\right)^{4}\right) c_{5}\right) e_{k}^{4} \\
& +\left(\gamma^{2} f^{\prime}\left(x^{*}\right)^{2}\left(4+3 \gamma f^{\prime}\left(x^{*}\right)\right) c_{2}^{2} c_{4}+\gamma f^{\prime}\left(x^{*}\right)(9\right. \\
& \left.+10 \gamma f^{\prime}\left(x^{*}\right)+3 \gamma^{2} f^{\prime}\left(x^{*}\right)^{2}\right) c_{3} c_{4}+\gamma f^{\prime}\left(x^{*}\right) \\
& \cdot c_{2}\left(2 \gamma f^{\prime}\left(x^{*}\right) c_{3}^{2}+\left(11+20 \gamma f^{\prime}\left(x^{*}\right)\right.\right. \\
& \left.\left.+15 \gamma^{2} f^{\prime}\left(x^{*}\right)^{2}+4 \gamma^{3} f^{\prime}\left(x^{*}\right)^{3}\right) c_{5}\right)+(6 \\
& +15 \gamma f^{\prime}\left(x^{*}\right)+20 \gamma^{2} f^{\prime}\left(x^{*}\right)^{2}+15 \gamma^{3} f^{\prime}\left(x^{*}\right)^{3} \\
& \left.\left.\left.+6 \gamma^{4} f^{\prime}\left(x^{*}\right)^{4}+\gamma^{5} f^{\prime}\left(x^{*}\right)^{5}\right) c_{6}\right) e_{k}^{5}\right]+O\left(e_{k}^{6}\right)
\end{aligned}
$$

Therefore, the error at the first step is

$$
\begin{aligned}
& y_{k}-x^{*}=\left(1+f^{\prime}\left(x^{*}\right) \gamma\right) c_{2} e_{k}^{2} \\
& +\left(-\left(2+2 f^{\prime}\left(x^{*}\right) \gamma+f^{\prime}\left(x^{*}\right)^{2} \gamma^{2}\right) c_{2}^{2}\right. \\
& \left.+\left(2+3 f^{\prime}\left(x^{*}\right) \gamma+f^{\prime}\left(x^{*}\right)^{2} \gamma^{2}\right) c_{3}\right) e_{k}^{3} \\
& +\left(\left(4+5 f^{\prime}\left(x^{*}\right) \gamma+3 f^{\prime}\left(x^{*}\right)^{2} \gamma^{2}+f^{\prime}\left(x^{*}\right)^{3} \gamma^{3}\right) c_{2}^{3}\right. \\
& -\left(7+10 f^{\prime}\left(x^{*}\right) \gamma+7 f^{\prime}\left(x^{*}\right)^{2} \gamma^{2}+2 f^{\prime}\left(x^{*}\right)^{3} \gamma^{3}\right) \\
& \cdot c_{2} c_{3} \\
& \left.+\left(3+6 f^{\prime}\left(x^{*}\right) \gamma+4 f^{\prime}\left(x^{*}\right)^{2} \gamma^{2}+f^{\prime}\left(x^{*}\right)^{3} \gamma^{3}\right) c_{4}\right) \\
& \cdot e_{k}^{4}+O\left(e_{k}^{5}\right) .
\end{aligned}
$$

By obtaining the Taylor expansion of $f\left(y_{k}\right)$ and $f\left[y_{k}, w_{k}\right]$, we get the following expression for the error equation: 


$$
\begin{aligned}
& e_{k+1}=\frac{\left(1+f^{\prime}\left(x^{*}\right) \gamma\right)(-1+\mu) c_{2}}{\mu} e_{k}^{2}+\frac{1}{\mu^{2}}((\beta(1 \\
& \left.+f^{\prime}\left(x^{*}\right) \gamma\right)^{2}-\mu\left(-3+\alpha\left(1+f^{\prime}\left(x^{*}\right) \gamma\right)^{2}\right. \\
& +2 f^{\prime}\left(x^{*}\right) \gamma(-2+\mu)+f^{\prime}\left(x^{*}\right)^{2} \gamma^{2}(-2+\mu) \\
& +2 \mu)) c_{2}^{2}+\left(2+3 f^{\prime}\left(x^{*}\right) \gamma+f^{\prime}\left(x^{*}\right)^{2} \gamma^{2}\right)(-1+\mu) \\
& \left.+\mu c_{3}\right) e_{k}^{3}+O\left(e_{k}^{4}\right) .
\end{aligned}
$$

It is clear that, imposing $\mu=1$, third-order is achieved and, then, the error equation is

$$
\begin{aligned}
& e_{k+1}=-(-1+\alpha-\beta)\left(1+f^{\prime}\left(x^{*}\right) \gamma\right)^{2} c_{2}^{2} e_{k}^{3}+((\alpha(1 \\
& \left.+f^{\prime}\left(x^{*}\right) \gamma\right)\left(6+7 f^{\prime}\left(x^{*}\right) \gamma+3 f^{\prime}\left(x^{*}\right)^{2} \gamma^{2}\right. \\
& \left.+\beta\left(1+f^{\prime}\left(x^{*}\right) \gamma\right)^{2}\right)-\left(1+f^{\prime}\left(x^{*}\right) \gamma\right)(3 \\
& +3 f^{\prime}\left(x^{*}\right) \gamma+2 f^{\prime}\left(x^{*}\right)^{2} \gamma^{2}+\left(\beta+f^{\prime}\left(x^{*}\right) \beta \gamma\right)^{2} \\
& \left.\left.+\beta\left(6+7 f^{\prime}\left(x^{*}\right) \gamma+3 f^{\prime}\left(x^{*}\right)^{2} \gamma^{2}\right)\right)\right) c_{2}^{3}-(1 \\
& \left.+f^{\prime}\left(x^{*}\right) \gamma\right)^{2}\left(-3-2 f^{\prime}\left(x^{*}\right) \gamma+2 \alpha\left(2+f^{\prime}\left(x^{*}\right) \gamma\right)\right. \\
& \left.\left.\quad-2 \beta\left(2+f^{\prime}\left(x^{*}\right) \gamma\right)\right) c_{2} c_{3}\right) e_{k}^{4}+O\left(e_{k}^{5}\right) .
\end{aligned}
$$

Again, setting $\beta=\alpha-1$, we get order four and

$$
\begin{aligned}
& e_{k+1}=\left(1+f^{\prime}\left(x^{*}\right) \gamma\right)^{2} c_{2}\left(\left(2+\alpha+f^{\prime}\left(x^{*}\right) \alpha \gamma\right) c_{2}^{2}\right. \\
& \left.-c_{3}\right) e_{k}^{4}-\left(1+f^{\prime}\left(x^{*}\right) \gamma\right)\left(\left(10+11 f^{\prime}\left(x^{*}\right) \gamma\right.\right. \\
& +5 f^{\prime}\left(x^{*}\right)^{2} \gamma^{2}+\alpha^{2}\left(1+f^{\prime}\left(x^{*}\right) \gamma\right)^{3}+\alpha(7 \\
& \left.\left.+13 f^{\prime}\left(x^{*}\right) \gamma+8 f^{\prime}\left(x^{*}\right)^{2} \gamma^{2}+2 f^{\prime}\left(x^{*}\right)^{3} \gamma^{3}\right)\right) c_{2}^{4} \\
& -\left(14+19 f^{\prime}\left(x^{*}\right) \gamma+7 f^{\prime}\left(x^{*}\right)^{2} \gamma^{2}+3 \alpha(1\right. \\
& \left.\left.+f^{\prime}\left(x^{*}\right) \gamma\right)^{2}\left(2+f^{\prime}\left(x^{*}\right) \gamma\right)\right) c_{2}^{2} c_{3}+(2 \\
& \left.+3 f^{\prime}\left(x^{*}\right) \gamma+f^{\prime}\left(x^{*}\right)^{2} \gamma^{2}\right) c_{3}^{2}+\left(2+3 f^{\prime}\left(x^{*}\right) \gamma\right. \\
& \left.\left.+f^{\prime}\left(x^{*}\right)^{2} \gamma^{2}\right) c_{2} c_{4}\right) e_{k}^{5}+O\left(e_{k}^{6}\right) .
\end{aligned}
$$

Let us remark not only that the fourth-order has been proven but also that factor $\left(1+f^{\prime}\left(x^{*}\right) \gamma\right)$ appears also in the term of order five.

Let us observe that this family of derivative-free methods (2) supports Kung-Traub's conjecture [6], having optimal efficiency index $I=4^{1 / 3} \approx 1.587$. On the other hand, by observing the expressions of the error equation (8) we can choose different values of the free disposable parameters in order to obtain iterative methods with memory, increasing the order of convergence.

\section{Iterative Methods with Memory}

We are going to design derivative-free schemes with memory based on the proposed methods (2).

From (8) we can assure that the order of convergence of family (2) increases up to six if $\gamma=-1 / f^{\prime}\left(x^{*}\right)$, but the value of $f^{\prime}\left(x^{*}\right)$ is not available in practice and such acceleration is not possible. However, we can use an approximation $\overline{f^{\prime}}\left(x^{*}\right) \approx$ $f^{\prime}\left(x^{*}\right)$, calculated by using known information. Therefore, by setting $\gamma=-1 / \overline{f^{\prime}}\left(x^{*}\right)$ we can increase the convergence order without using new functional evaluations. The main idea in constructing methods with memory consists of the calculation of the parameter $\gamma=\gamma_{k}$ as the iteration proceeds by $\gamma_{k}=-1 / \overline{f^{\prime}}\left(x^{*}\right), k=1,2, \ldots$ We are going to consider different approximations of $f^{\prime}\left(x^{*}\right)$.

(1) Let $N_{1}(t)=N_{1}\left(t, x_{k}, x_{k-1}\right)$ be Newton's interpolation polynomial of first degree through two available approximations $x_{k}, x_{k-1}$; that is $N_{1}(t)=f\left(x_{k}\right)+f\left[x_{k}, x_{k-1}\right]\left(t-x_{k}\right)$, so

$$
\gamma_{k}=\frac{-1}{N_{1}^{\prime}\left(x_{k}\right)}=\frac{-1}{f\left[x_{k}, x_{k-1}\right]}=-\frac{x_{k}-x_{k-1}}{f\left(x_{k}\right)-f\left(x_{k-1}\right)},
$$

and the algorithm denoted by $\operatorname{MM1}(\alpha)$ can be presented in the following way:

(i) $x_{0}, \gamma_{0}$ are given.

(ii) $w_{k}=x_{k}+\gamma_{k} f\left(x_{k}\right), k=0,1,2, \ldots$

(iii) $y_{k}=x_{k}-f\left(x_{k}\right) / f\left[x_{k}, w_{k}\right]$.

(iv) $x_{k+1}=y_{k}-\left(\left(f\left(x_{k}\right)+\alpha f\left(y_{k}\right)\right) /\left(f\left(x_{k}\right)+(\alpha-\right.\right.$ 1) $\left.\left.f\left(y_{k}\right)\right)\right)\left(f\left(y_{k}\right) / f\left[y_{k}, w_{k}\right]\right)$,

where $\gamma_{k}=-1 / f\left[x_{k}, x_{k-1}\right]$ and $\alpha$ is a free parameter.

The order of convergence of this algorithm is analyzed in the following result.

Theorem 2. Let $x^{*}$ be a simple zero of a sufficiently differentiable function $f: I \subseteq \mathbb{R} \rightarrow \mathbb{R}$ in an open interval I. If $x_{0}$ is close enough to $x^{*}$ and $\gamma_{0}$ is given, then the R-order of family $M M 1(\alpha)$ is at least $2+\sqrt{6} \approx 4.45$ that corresponds to the positive root of polynomial $p^{2}-4 p-2$.

Proof. By expanding in Taylor series $f\left(x_{k}\right)$ and $f\left(x_{k-1}\right)$, we get

$$
\begin{aligned}
& f\left(x_{k}\right)=f^{\prime}\left(x^{*}\right) \\
& \cdot\left(e_{k}+c_{2} e_{k}^{2}+c_{3} e_{k}^{3}+c_{4} e_{k}^{4}+c_{5} e_{k}^{5}+c_{6} e_{k}^{6}\right)+O\left(e_{k}^{7}\right), \\
& f\left(x_{k-1}\right)=f^{\prime}\left(x^{*}\right) \\
& \quad \cdot\left(e_{k-1}+c_{2} e_{k-1}^{2}+c_{3} e_{k-1}^{3}+c_{4} e_{k-1}^{4}+c_{5} e_{k-1}^{5}+c_{6} e_{k-1}^{6}\right) \\
& \quad+O\left(e_{k-1}^{7}\right) .
\end{aligned}
$$


Then,

$$
\begin{aligned}
\gamma_{k} & =-\frac{1}{f\left[x_{k}, x_{k-1}\right]}=\frac{1}{f^{\prime}\left(x^{*}\right)}\left[-1+c_{2}\left(e_{k}+e_{k-1}\right)\right. \\
& +\left(-2 c_{2}^{2}+c_{3}\right) e_{k} e_{k-1}+\left(-c_{2}^{2}+c_{3}\right)\left(e_{k}^{2}+e_{k-1}^{2}\right) \\
& +\left(3 c_{2}^{3}-4 c_{2} c_{3}+c_{4}\right)\left(e_{k}^{2} e_{k-1}+e_{k} e_{k-1}^{2}\right)+\left(c_{2}^{3}-2 c_{2} c_{3}\right. \\
& \left.\left.+c_{4}\right)\left(e_{k}^{3}+e_{k-1}^{3}\right)\right]+O_{4}\left(e_{k}, e_{k-1}\right), \\
w_{k} & -x^{*}=e_{k} e_{k-1}\left[\left(c_{2}^{5}-4 c_{3} c_{2}^{3}+3 c_{4} c_{2}^{2}+\left(3 c_{3}^{2}-2 c_{5}\right) c_{2}\right.\right. \\
& \left.-2 c_{3} c_{4}+c_{6}\right) e_{k-1}^{4}-\left(c_{2}^{4}-3 c_{3} c_{2}^{2}+2 c_{4} c_{2}+c_{3}^{2}-c_{5}\right) e_{k-1}^{3} \\
& \left.+\left(c_{2}^{3}-2 c_{3} c_{2}+c_{4}\right) e_{k-1}^{2}+\left(c_{3}-c_{2}^{2}\right) e_{k-1}+c_{2}\right] \\
& +e_{k}^{2} e_{k-1}\left[\left(4 c_{2}^{5}-13 c_{3} c_{2}^{3}+7 c_{4} c_{2}^{2}+\left(8 c_{3}^{2}-3 c_{5}\right) c_{2}\right.\right. \\
& \left.-4 c_{3} c_{4}+c_{6}\right) e_{k-1}^{3}+\left(-3 c_{2}^{4}+7 c_{3} c_{2}^{2}-3 c_{4} c_{2}-2 c_{3}^{2}\right. \\
& \left.\left.+c_{5}\right) e_{k-1}^{2}+\left(2 c_{2}^{3}-3 c_{3} c_{2}+c_{4}\right) e_{k-1}-c_{2}^{2}+c_{3}\right] \\
& +O_{4}\left(e_{k}, e_{k-1}\right),
\end{aligned}
$$

where $O_{4}\left(e_{k}, e_{k-1}\right)$ indicates that the sum of the exponents of $e_{k-1}$ and $e_{k}$ in the rejected terms of the development is at least 4.

The error at the next step is

$$
\begin{aligned}
y_{k} & -x^{*}=c_{2} e_{k}^{2} e_{k-1}\left[c_{2}+\left(c_{3}-c_{2}^{2}\right) e_{k-1}\right. \\
& +\left(c_{2}^{3}-2 c_{3} c_{2}+c_{4}\right) e_{k-1}^{2} \\
& \left.-\left(c_{2}^{4}-3 c_{3} c_{2}^{2}+2 c_{4} c_{2}+c_{3}^{2}-c_{5}\right) e_{k-1}^{3}\right] \\
& +e_{k}^{3} e_{k-1}\left[\left(-2 c_{2}^{3}+2 c_{2} c_{3}\right)\right. \\
& +\left(2 c_{2}^{4}-4 c_{2}^{2} c_{3}+c_{3}^{2}+c_{2} c_{4}\right) e_{k-1} \\
& +\left(-2 c_{2}^{5}+6 c_{2}^{3} c_{3}-4 c_{2}^{2} c_{4}+c_{3} c_{4}+c_{2}\left(-2 c_{3}^{2}+c_{5}\right)\right) \\
& \left.\cdot e_{k-1}^{2}\right]+O_{5}\left(e_{k}, e_{k-1}\right)
\end{aligned}
$$

and, then,

$$
\begin{aligned}
& \frac{f\left(x_{k}\right)}{f\left[y_{k}, w_{k}\right]}=\left[c_{2}^{2} e_{k-1}+\left(-c_{2}^{3}+c_{2} c_{3}\right) e_{k-1}^{2}\right. \\
& \left.\quad+c_{2}\left(c_{2}^{3}-2 c_{2} c_{3}+c_{4}\right) e_{k-1}^{3}\right] e_{k}^{2} \\
& \quad+\left[\left(-2 c_{2}^{3}+2 c_{2} c_{3}\right) e_{k-1}\right. \\
& \left.\quad+\left(c_{2}^{4}-4 c_{2}^{2} c_{3}+c_{3}^{2}+c_{2} c_{4}\right) e_{k-1}^{2}\right] e_{k}^{3}+O_{5}\left(e_{k}, e_{k-1}\right)
\end{aligned}
$$

Moreover,

$$
\begin{aligned}
& \frac{f\left(x_{k}\right)+\alpha f\left(y_{k}\right)}{f\left(x_{k}\right)+(\alpha-1) f\left(y_{k}\right)}=\left[c_{2}^{2} e_{k-1}+\left(-c_{2}^{3}+c_{2} c_{3}\right) e_{k-1}^{2}\right. \\
& \left.\quad+c_{2}\left(c_{2}^{3}-2 c_{2} c_{3}+c_{4}\right) e_{k-1}^{3}\right] e_{k} \\
& \quad+\left[\left(-3 c_{2}^{3}+2 c_{2} c_{3}\right) e_{k-1}\right. \\
& \left.\quad+\left(-(-4+\alpha) c_{2}^{4}-5 c_{2}^{2} c_{3}+c_{3}^{2}+c_{2} c_{4}\right) e_{k-1}^{2}\right] e_{k}^{2} \\
& \quad+\left[\left(6 c_{2}^{4}-9 c_{2}^{2} c_{3}+c_{3}^{2}+2 c_{2} c_{4}\right) e_{k-1}^{3}\right] e_{k}^{3} \\
& \quad+O_{5}\left(e_{k}, e_{k-1}\right) .
\end{aligned}
$$

Finally, by combining the previous expansions from (12)-(14),

$$
e_{k+1}=\left(2 c_{2}^{5}-c_{2}^{3} c_{3}\right) e_{k}^{4} e_{k-1}^{2}+O_{6}\left(e_{k}, e_{k-1}\right) .
$$

Since the lower term of the error equation is $\left(2 c_{2}^{5}-c_{2}^{3} c_{3}\right) e_{k-1}^{2} e_{k}^{4}$, applying Theorem 9.2.9 of [12], the powers of $e_{k}$ and $e_{k-1}$ are 4 and 2 , respectively, so the polynomial whose real roots give the $R$-order of the method is $p^{2}-4 p+2$ and the order of the method is, at least, $2+\sqrt{6}$.

(2) Let $N_{2}(t)=N_{2}\left(t, x_{k}, x_{k-1}, y_{k-1}\right)$ (can be also used as $\left.N_{2}(t)=N_{2}\left(t, x_{k}, x_{k-1}, w_{k-1}\right)\right)$ be Newton's interpolation polynomial of second degree; that is, $N_{2}(t)=f\left(x_{k}\right)+$ $f\left[x_{k}, x_{k-1}\right]\left(t-x_{k}\right)+f\left[x_{k}, x_{k-1}, y_{k-1}\right]\left(t-x_{k}\right)\left(t-x_{k-1}\right)$, and therefore

$$
\begin{aligned}
\gamma_{k} & =\frac{-1}{N_{2}^{\prime}\left(x_{k}\right)} \\
& =\frac{-1}{f\left[x_{k}, x_{k-1}\right]+f\left[x_{k}, x_{k-1}, y_{k-1}\right]\left(x_{k}-x_{k-1}\right)},
\end{aligned}
$$

and a similar algorithm to the previous one, denoted by $\operatorname{MM} 2(\alpha)$, can be presented. The convergence of the method is established in the following result, whose proof is similar to that of the last proposed method. This is the reason why it is avoided.

Theorem 3. Let $x^{*}$ be a simple zero of a sufficiently differentiable function $f: I \subseteq \mathbb{R} \rightarrow \mathbb{R}$ in an open interval I. If $x_{0}$ is close enough to $x^{*}$ and $\gamma_{0}$ is given, then the R-order of family MM2 is at least 5.

(3) Let $N_{3}(t)=N_{3}\left(t, x_{k}, x_{k-1}, y_{k-1}, w_{k-1}\right)$ be Newton's interpolation polynomial of third degree: $N_{3}(t)=f\left(x_{k}\right)+$ $f\left[x_{k}, x_{k-1}\right]\left(t-x_{k}\right)+f\left[x_{k}, x_{k-1}, y_{k-1}\right]\left(t-x_{k}\right)\left(t-x_{k-1}\right)+$ $f\left[x_{k}, x_{k-1}, y_{k-1}, w_{k-1}\right]\left(t-x_{k}\right)\left(t-x_{k-1}\right)\left(t-y_{k-1}\right)$, and then

$$
\begin{aligned}
& N_{3}^{\prime}\left(x_{k}\right) \\
& =f\left[x_{k}, x_{k-1}\right]+f\left[x_{k}, x_{k-1}, y_{k-1}\right]\left(x_{k}-x_{k-1}\right) \\
& \quad+f\left[x_{k}, x_{k-1}, y_{k-1}, w_{k-1}\right]\left(x_{k}-x_{k-1}\right)\left(x_{k}-y_{k-1}\right), \\
& \gamma_{k}=\frac{-1}{N_{3}^{\prime}\left(x_{k}\right)} .
\end{aligned}
$$


The algorithm denoted by $\operatorname{MM} 3(\alpha)$ can be presented in the following way:

(i) $x_{0}, \gamma_{0}$ are given.

(ii) $w_{k}=x_{k}+\gamma_{k} f\left(x_{k}\right), k=0,1,2, \ldots$.

(iii) $y_{k}=x_{k}-f\left(x_{k}\right) / f\left[x_{k}, w_{k}\right]$.

(iv) $x_{k+1}=y_{k}-\left(\left(f\left(x_{k}\right)+\alpha f\left(y_{k}\right)\right) /\left(f\left(x_{k}\right)+(\alpha-\right.\right.$ 1) $\left.\left.f\left(y_{k}\right)\right)\right)\left(f\left(x_{k}\right) / f\left[y_{k}, w_{k}\right]\right)$,

where $\gamma_{k}=-1 / N_{3}^{\prime}\left(x_{k}\right)$ and $\alpha$ is a free parameter.

Theorem 4. Let $x^{*}$ be a simple zero of a sufficiently differentiable function $f: I \subseteq \mathbb{R} \rightarrow \mathbb{R}$ in an open interval I. If $x_{0}$ is close enough to $x^{*}$ and $\gamma_{0}$ is given, then the R-order of family MM3 is at least 6.

Proof. By using Taylor expansion around $x^{*}$ of the different elements of $N_{3}^{\prime}\left(x_{k}\right)$, we prove that

$$
\begin{aligned}
1+\gamma_{k} f^{\prime}\left(x^{*}\right) & =1-\frac{f^{\prime}\left(x^{*}\right)}{N_{3}^{\prime}(x)} \sim c_{4} e_{k-1} e_{y, k-1} e_{w, k-1} \\
& \sim e_{k-1} e_{y, k-1} e_{w, k-1},
\end{aligned}
$$

where $e_{k}=x_{k}-x^{*}, e_{y, k}=y_{k}-x^{*}$, and $e_{w, k}=w_{k}-x^{*}$.

Let $\left\{x_{k}\right\}$ be the sequence generated by the algorithm MM3. If $\left\{x_{k}\right\}$ converges to $x^{*}$ with $R$-order $r$, then

$$
e_{k+1} \sim D_{k, r} e_{k}^{r},
$$

such that $\lim _{k \rightarrow \infty} D_{k, r}=D_{r}$, with this limit being the asymptotic error constant of the method. So,

$$
e_{k+1} \sim D_{k, r}\left(D_{k-1, r} e_{k-1}^{r}\right)^{r}=D_{k, r} D_{k-1, r}^{r} e_{k-1}^{r^{2}} .
$$

Let us suppose that $\left\{w_{k}\right\}$ has $R$-order $r_{1}$, and then

$$
e_{w, k} \sim D_{k, r_{1}} e_{k}^{r_{1}} \sim D_{k, r_{1}} D_{k-1, r}^{r} e_{k-1}^{r r_{1}} .
$$

Analogously, if sequence $\left\{y_{k}\right\}$ has $R$-order $r_{2}$, we obtain

$$
e_{y, k} \sim D_{k, r_{2}} D_{k-1, r}^{r} e_{k-1}^{r r_{2}} \text {. }
$$

On the other hand, we obtain the error equation of the different sequences without memory

$$
e_{k+1}=\left(1+\gamma f^{\prime}\left(x^{*}\right)\right)^{2} M e_{k}^{4}+O\left(e_{k}^{5}\right),
$$

where $M=c_{2}\left(2+\alpha\left(1+\gamma f^{\prime}\left(x^{*}\right)\right) c_{2}^{2}-c_{3}\right)$ and

$$
\begin{aligned}
& e_{w, k}=\left(1+\gamma f^{\prime}\left(x^{*}\right)\right) e_{k}+O\left(e_{k}^{2}\right), \\
& e_{y, k}=c_{2}\left(1+\gamma f^{\prime}\left(x^{*}\right)\right) e_{k}^{2}+O\left(e_{k}^{3}\right) .
\end{aligned}
$$

Therefore, the corresponding error relations with memory are

$$
\begin{aligned}
& e_{k+1} \sim\left(1+\gamma f^{\prime}\left(x^{*}\right)\right)^{2} M_{k} e_{k}^{4}, \\
& e_{w, k} \sim\left(1+\gamma f^{\prime}\left(x^{*}\right)\right) e_{k}, \\
& e_{y, k} \sim c_{2}\left(1+\gamma f^{\prime}\left(x^{*}\right)\right) e_{k}^{2},
\end{aligned}
$$

and, by using expression (18) and simple algebraic manipulations, we obtain

$$
\begin{aligned}
e_{k+1} & \sim D_{k-1, r_{1}}^{2} D_{k-1, r_{2}}^{2} D_{k-1, r}^{4} e_{k-1}^{4 r+2 r_{1}+2 r_{2}+2}, \\
e_{w, k} & \sim D_{k-1, r_{1}} D_{k-1, r_{2}} D_{k-1, r} e_{k-1}^{r+r_{1}+r_{2}+1}, \\
e_{y, k} & \sim c_{2} D_{k-1, r_{1}} D_{k-1, r_{2}} D_{k-1, r}^{2} e_{k-1}^{2 r+r_{1}+r_{2}+1} .
\end{aligned}
$$

If we compare the exponents of $e_{k-1}$ between "(20), (21), (22), and (26)), ((21), (22), (26), and (27)" and "(22), (26), (27), and (28)," we obtain the following nonlinear system:

$$
\begin{aligned}
& r^{2}=4 r+2 r_{1}+2 r_{2}+2, \\
& r r_{1}=r+r_{1}+r_{2}+1, \\
& r r_{2}=2 r+r_{1}+r_{2}+1 .
\end{aligned}
$$

It is easy to obtain the unique real solution of this system: $r_{1}=2, r_{2}=3$, and $r=6$. Therefore, the $R$-order of algorithm MM3 is 6 for any value of parameter $\alpha$.

Many other approximations of $f^{\prime}\left(x^{*}\right)$ are possible, but they either are of sixth order (with more computational cost) or are lower than six.

\section{Real Dynamics}

In this section, the real dynamics of the iterative family are analyzed. After an introduction of real dynamics fundamentals $[13,14]$, the features of the family are described when it is applied over the nonlinear polynomials $x^{2}-1, x^{2}$, and $x^{2}+1$.

4.1. Fundamentals on Real Dynamics. Let $M: \mathbb{R} \rightarrow \mathbb{R}$ be a rational function. The orbit of a point $x_{0} \in \mathbb{R}$ is defined as the set

$$
\left\{x_{0}, M\left(x_{0}\right), M^{2}\left(x_{0}\right), \ldots, M^{n}\left(x_{0}\right), \ldots\right\} .
$$

A point $x_{0}$ is a fixed point, $x_{0}^{F}$, of $M$ if $M\left(x_{0}^{F}\right)=x_{0}^{F}$. The multiplier $\left|M^{\prime}\left(x_{0}^{F}\right)\right|$ classifies the fixed points as attracting, repelling, or neutral if its value is lower than, greater than, or equal to 1 , respectively. When the multiplier is zero, the fixed point is superattracting. Both attracting and superattracting fixed points are denoted as $x^{*}$.

The character of the infinity is set by the study of $I(x)=$ $1 / M(1 / x)[15]$. The point $\infty$ is a fixed point of $M$ if and only if $x=0$ is a fixed point of $I$. In this case, the multiplier of $\infty$ is $\left|I^{\prime}(0)\right|$. When this value is zero, the point $\infty$ is a superattracting point.

The basin of attraction of an attracting fixed point $x^{*}$, $\mathscr{A}\left(x^{*}\right)$, is defined as the set of preimages of any order such that

$$
\mathscr{A}\left(x^{*}\right)=\left\{x_{0} \in \mathbb{R}: M^{n}\left(x_{0}\right) \longrightarrow x^{*}, n \longrightarrow \infty\right\} .
$$

The Fatou set, $\mathscr{F}(M)$, includes the points whose orbits tend to an attracting point $x^{*}$. The Julia set, $\mathscr{F}(M)$, is its complementary. It covers the repelling points and sets the borders between the basins of attraction. 


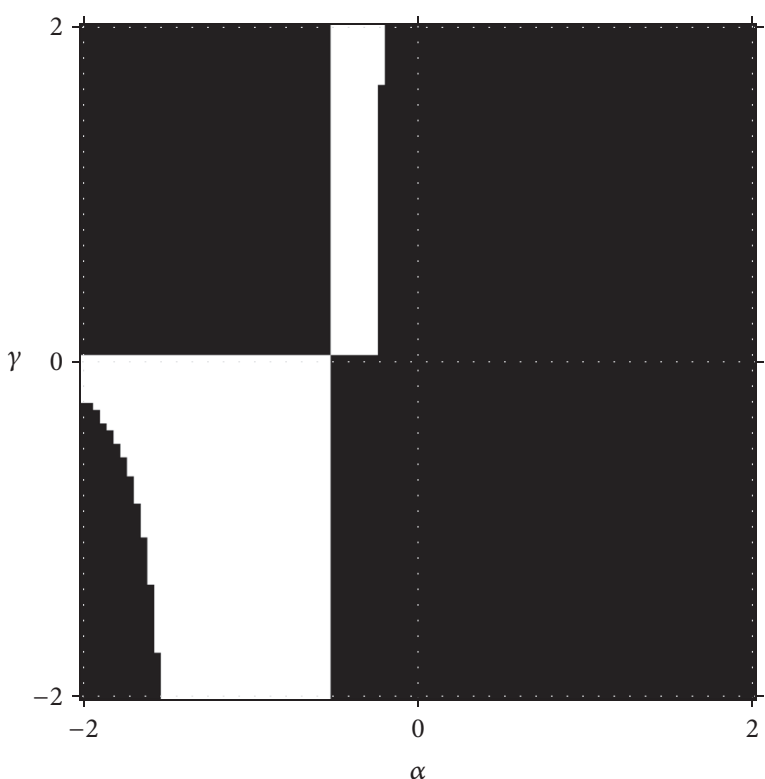

(a)

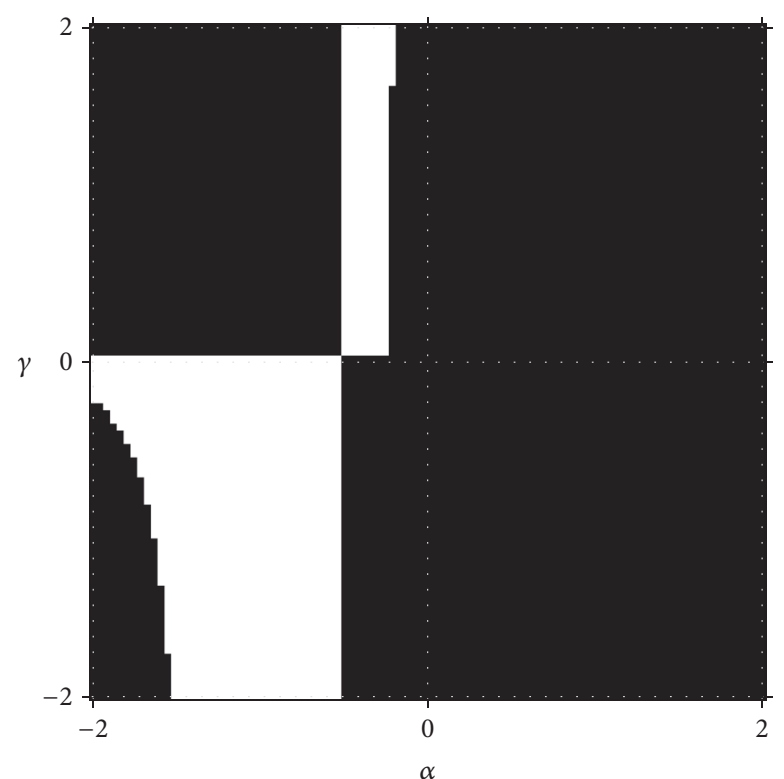

(b)

FIGURE 1: Stability plane of (a) $x_{3}^{F}$ and (b) $x_{4}^{F}$.

The fixed point operator of the biparametric family (2) is

$$
M_{f}(x)=y-\frac{f(x)+\alpha f(y)}{f(x)+(\alpha-1) f(y)} \frac{f(y)}{f[y, w]},
$$

where $w=x+\gamma f(x)$ and $y=x-f(x) / f[x, w]$.

4.2. Application on Quadratic Polynomials. We are going to analyze the dynamical behavior of the rational function obtained when family (32) is applied on $f(x) \in\left\{x^{2}-\right.$ $\left.1, x^{2}, x^{2}+1\right\}$.

4.2.1. $f(x)=x^{2}-1$. In this case, expression (32) is $M_{f}(x)=$ $N_{11}(x) / D_{10}(x)$, where $N_{11}(x)$ and $D_{10}(x)$ are polynomials of degrees 11 and 10, respectively, depending on $x$ and on parameters $\alpha$ and $\gamma$.

There are 10 fixed points of $M_{f}(x) \cdot x_{1,2}^{F}=x_{1,2}^{*}= \pm 1$ are superattracting points, while $x_{3-10}^{F}(\alpha, \gamma)$ are the roots of an 8th-degree polynomial: $x_{3,4}^{F} \in \mathbb{R}$ and $x_{5-10}^{F} \in \mathbb{C}$. Since our purpose is the study of the real dynamics, $x_{5-10}^{F}$ are rejected. The evaluation of $\left|M_{f}^{\prime}\left(x_{3,4}^{F}\right)\right|$ establishes the behavior of these points. For different values of $\alpha$ and $\gamma, x_{3,4}^{F}$ have different dynamical features, as the stability plane of Figure 1 represents. A mesh of $100 \times 100$ points covers the values of $\alpha \in[-2,2]$ and $\gamma \in[-2,2]$. The white area represents where the multiplier is lower than 1 , while the corresponding black represents where the multiplier is greater than 1 . Its dynamical meaning is immediate, since white and black regions represent attracting and repelling behavior, respectively.

Therefore, $x_{3}^{F}$ has both behaviors, depending on the value of $\alpha$ and $\gamma$. For assuring the convergence of the method to $x_{1,2}^{*}$, white areas must be avoided.
The point $\infty$ is a parabolic fixed point, since $I(0)=0$ and $I^{\prime}(0)=1$.

Computing $M_{f}^{\prime}(x)=0,16$ critical points can be found. As expected, $x_{1,2}^{C}=x_{1,2}^{*} \cdot x_{3,4}^{C}=(-1 \pm \gamma) / \gamma$ are preimages of $x_{1,2}^{*} . x_{5-16}^{C}$ are the roots of a polynomial of degree 12 , that depend on the parameters $\alpha$ and $\gamma$. Six of these roots are real $\left(x_{5-10}^{C}\right)$ while the other ones are complex $\left(x_{11-16}^{C}\right)$.

A classical result (attributed to Fatou and Julia) establishes that there is at least one critical point associated with each invariant Fatou component, so it is interesting to analyze the behavior of each free critical point (critical point different to the roots of the polynomial) used as initial estimation for all the elements of the family.

Just as the convergence plane [4] represents the final state of the orbits of every starting point in a single-parametric family of methods, we use the parameters plane to guess the final state of the orbit of one critical point in a biparametric family of methods, in a similar manner as [16]. Therefore, the parameters plane represents the family of methods $M_{f}(x)$, where each point $(\alpha, \gamma)$ stands for an individual method. If the orbit of the free critical point $x^{C}$ tends to $-1,1$, or $\infty$, the point is colored in red, green, or blue, respectively. If $x^{C}$ does not converge to any of those points, the point is colored in black. Figure 2 shows the parameter planes of $x_{5-10}^{C}$.

The unified parameter plane represented in Figure 3 gathers in one image the main information of Figure 2. It is composed of the superposition of the black regions. In this way, the election of a point in the white region guarantees that the corresponding scheme tends to \pm 1 or $\infty$, while the set of methods in the black region do not.

In order to visualize the behavior of each method, a new representation concept is introduced: the dynamical line. 


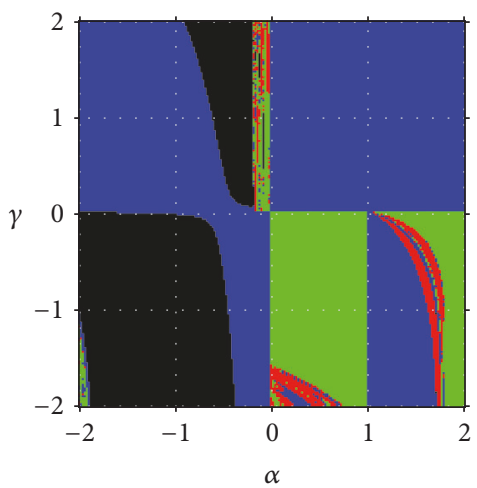

(a)

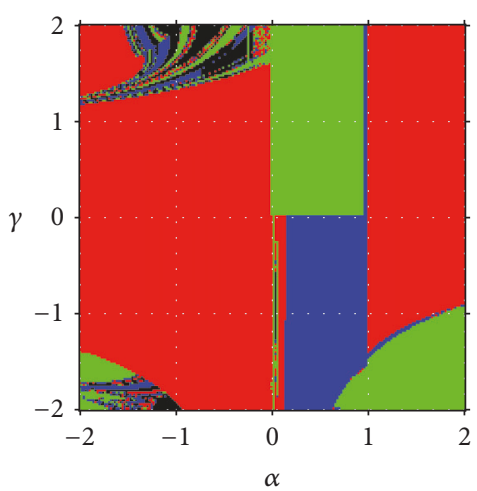

(d)

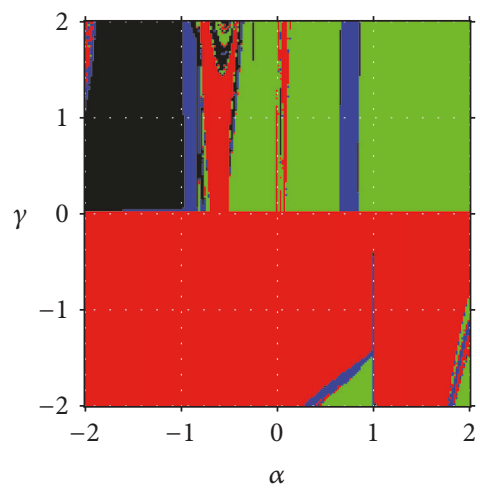

(b)

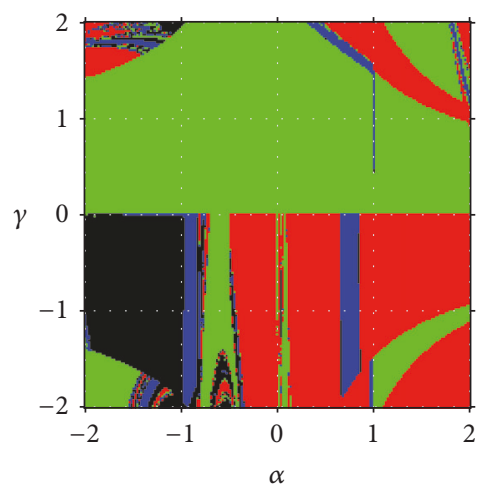

(e)

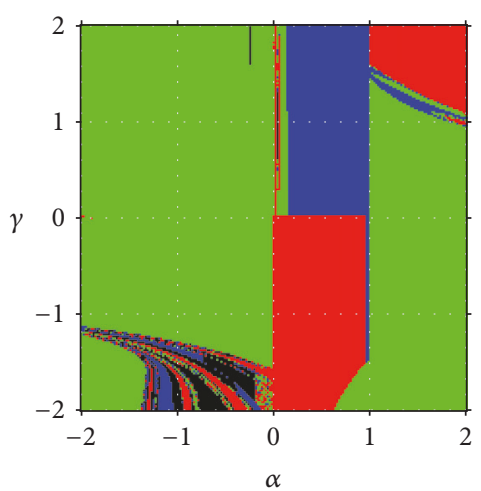

(c)

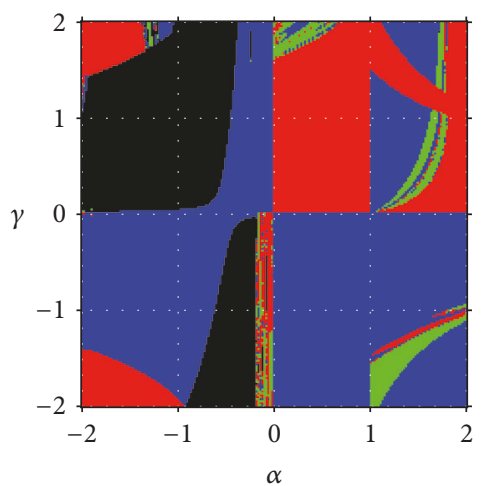

(f)

Figure 2: Parameter planes of (a) $x_{5}^{C}$, (b) $x_{6}^{C}$, (c) $x_{7}^{C}$, (d) $x_{8}^{C}$, (e) $x_{9}^{C}$, and (f) $x_{10}^{C}$ (red: $x_{1}^{*}$, green: $x_{2}^{*}$, blue: $x_{\infty}^{*}$, and black: others).

Analogous to the dynamical plane for complex variable, the dynamical line represents the basins of attraction, plotting in different colors where the orbit of each initial estimation tends. In coherence to Figure 2, the red color is assigned to those initial approximations that tend to $x_{1}^{*}=-1$. Analogously, the green color is assigned to $x_{2}^{*}=1$, the blue color is assigned to $x^{*}=\infty$, and the black one is assigned to those initial guesses that tend to a point different than the previous three. The final orbit of 1001 initial values of $x \in[-2,2]$ has been calculated, and the convergence to a point is set when its distance is lower than $10^{-3}$.

Figure 4 represents the dynamical line of a set of methods whose orbits tend to $-1,1$, or $\infty$, that is, methods that belong to the white area of the unified parameter plane. On the contrary, Figure 5 shows methods whose points also tend to another fixed point.

Note that every point in Figure 4 tends to one of the attracting points. However, when a value of $(\alpha, \gamma)$ of the black region of Figure 3 is chosen, some points tend to a different point. For instance, these points can be found in Figures $5(\mathrm{a})-5(\mathrm{~d})$ for $x_{0}=-0.2, x_{0}=0.2, x_{0}=-0.28$, and $x_{0}=0.28$, respectively.

As expected from the attracting area of $x_{3}^{F}$ and the unified parameter plane, the election of methods with $\alpha>0$ is capital, as proved in Figures 4 and 5.
4.2.2. $f(x)=x^{2}$. Applying (32) over $f(x)=x^{2}$, the fixed point operator is

$$
\begin{aligned}
& M(x) \\
& =\frac{x(1+\gamma x)\left(5+5 \gamma x+\gamma^{2} x^{2}+\alpha(2+\gamma x)(1+\gamma x)^{2}\right)}{(2+\gamma x)(3+\gamma x)\left(3+2 \gamma x+\alpha\left(1+\gamma x^{2}\right)\right)} .
\end{aligned}
$$

The real fixed points of the operator are $x_{1}^{F}=0$ and $x_{2}^{F}(\alpha, \gamma)$, while the other two fixed points are complex. Both real fixed points have a different dynamical behavior depending on the parameter $\alpha . x_{1}^{F}$ is attracting for every value of $\alpha$ but the interval $-3.25<\alpha<-2.875 . x_{2}^{F}$ is repelling for every value of $\alpha \in(-3.25,-0.098)$. The stability planes of Figure 6 illustrate this behavior. Consequently, for

(i) $\alpha \in(-\infty,-3.25) \cup(-0.098,+\infty), x_{1}^{F}=x_{1}^{*}$ is attracting, and $x_{2}^{F}$ is repelling,

(ii) $\alpha \in(-3.25,-2.875), x_{1}^{F}$ is repelling, and $x_{2}^{F}=x_{2}^{*}$ is attracting,

(iii) $\alpha \in(-2.875,-0.098), x_{1}^{F}=x_{1}^{*}$, and $x_{2}^{F}=x_{2}^{*}$ are attracting.

From a stability point of view, just the first set of values of $\alpha$ cause the family to have a good performance, since the only attracting point is the root of $f(x)$. 


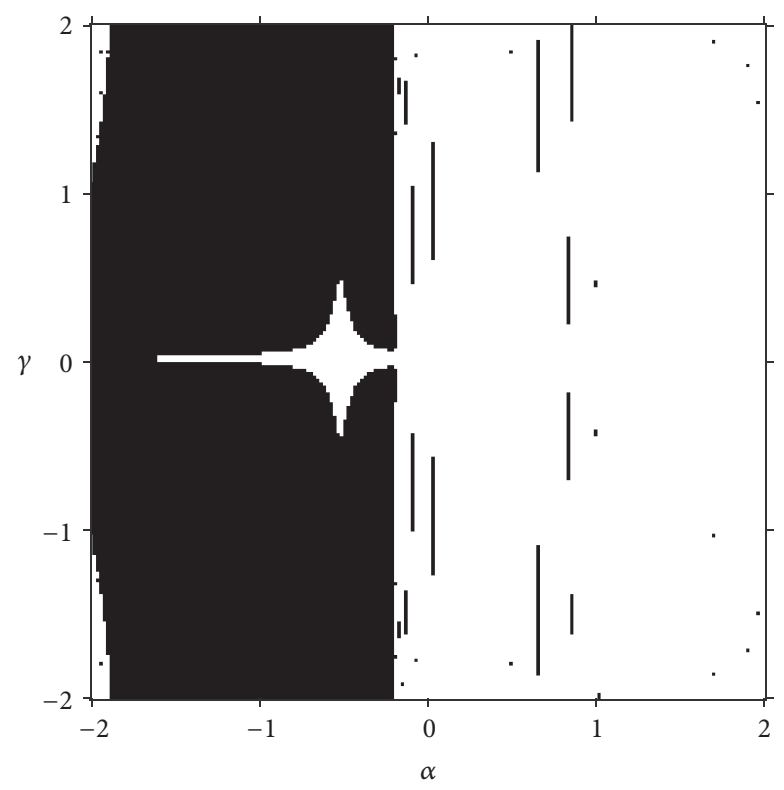

FIgURE 3: Unified parameter plane of $x_{5-10}^{C}$

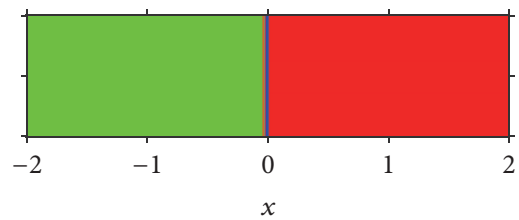

(a)

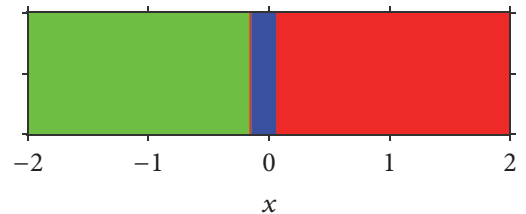

(d)

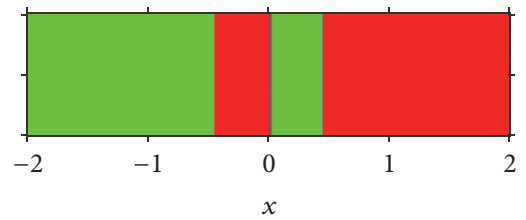

(g)

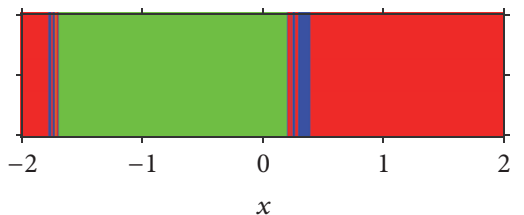

(b)

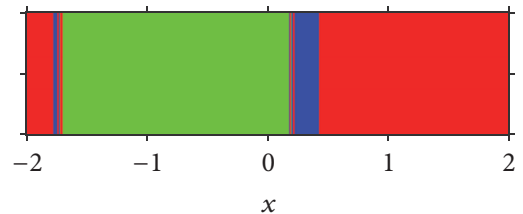

(e)

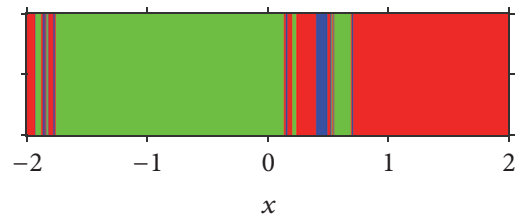

(h)

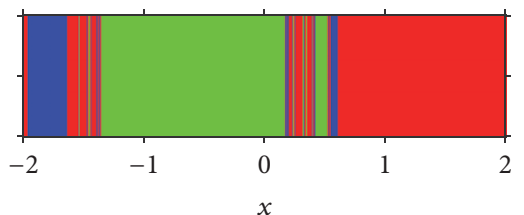

(c)

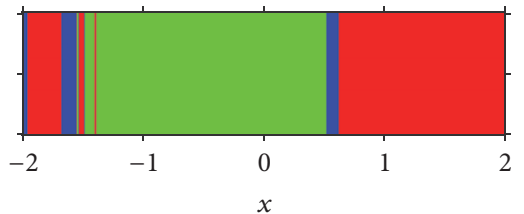

(f)

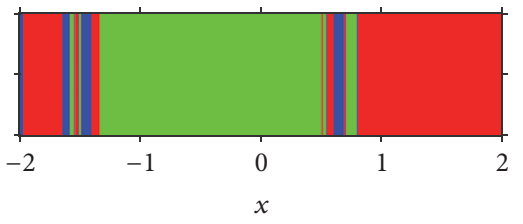

(i)

Figure 4: Dynamical lines of methods with (a) $(\alpha, \gamma)=(0.5,0.05),(\mathrm{b})(\alpha, \gamma)=(0.5,1),(\mathrm{c})(\alpha, \gamma)=(0.5,2),(\mathrm{d})(\alpha, \gamma)=(1,0.05),(\mathrm{e})$ $(\alpha, \gamma)=(1,1),(\mathrm{f})(\alpha, \gamma)=(1,2),(\mathrm{g})(\alpha, \gamma)=(2,0.05),(\mathrm{h})(\alpha, \gamma)=(2,1)$, and (i) $(\alpha, \gamma)=(2,2)$ (red: $x_{1}^{*}$, green: $x_{2}^{*}$, and blue: $\left.x_{\infty}^{*}\right)$.

In a similar fashion as the case $f(x)=x^{2}-1$, the infinity is a parabolic fixed point.

The zero values of the derivative of (33) are the critical points, which correspond to the roots of polynomial

$$
\begin{aligned}
& x^{8} \alpha^{2} \gamma^{8}+x^{7}\left(14 \alpha^{2} \gamma^{7}+4 \alpha \gamma^{7}\right) \\
& +x^{6}\left(77 \alpha^{2} \gamma^{6}+50 \alpha \gamma^{6}+2 \gamma^{6}\right) \\
& +x^{5}\left(224 \alpha^{2} \gamma^{5}+252 \alpha \gamma^{5}+26 \gamma^{5}\right)
\end{aligned}
$$

$$
\begin{aligned}
& +x^{4}\left(383 \alpha^{2} \gamma^{4}+666 \alpha \gamma^{4}+139 \gamma^{4}\right) \\
& +x^{3}\left(398 \alpha^{2} \gamma^{3}+996 \alpha \gamma^{3}+376 \gamma^{3}\right) \\
& +x^{2}\left(247 \alpha^{2} \gamma^{2}+842 \alpha \gamma^{2}+529 \gamma^{2}\right) \\
& +x\left(84 \alpha^{2} \gamma+372 \alpha \gamma+360 \gamma\right)+12 \alpha^{2}+66 \alpha+90
\end{aligned}
$$




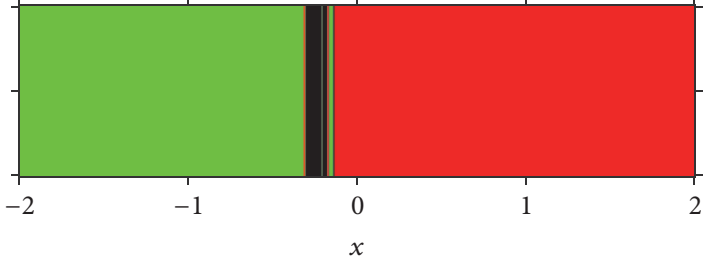

(a)

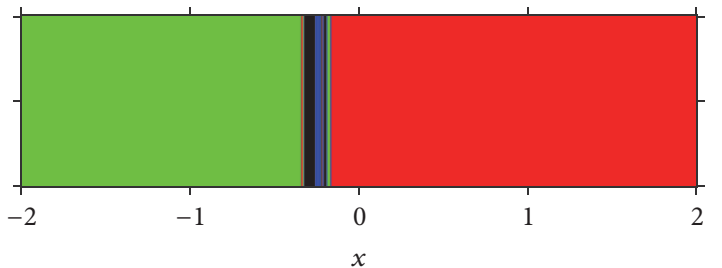

(c)

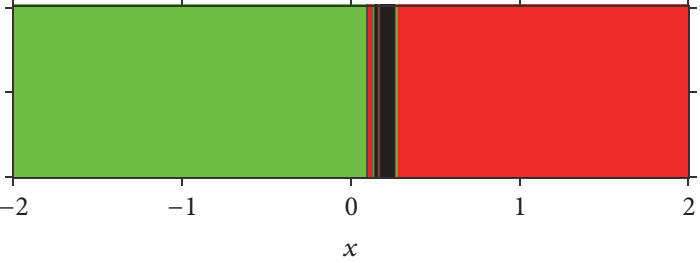

(b)

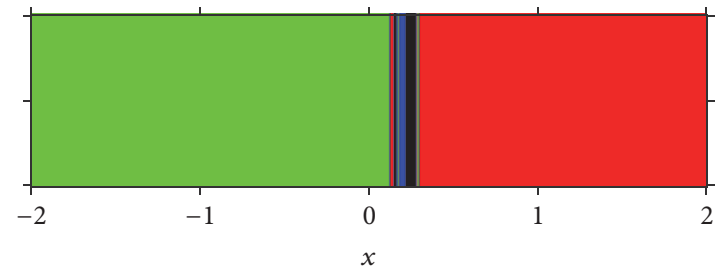

(d)

Figure 5: Dynamical lines of methods with (a) $(\alpha, \gamma)=(-0.5,-0.5)$, (b) $(\alpha, \gamma)=(-0.5,0.5),(\mathrm{c})(\alpha, \gamma)=(-1,-0.5)$, and $(\mathrm{d})(\alpha, \gamma)=(-1,0.5)$ (red: $x_{1}^{*}$, green: $x_{2}^{*}$, blue: $x_{\infty}^{*}$, and black: others).

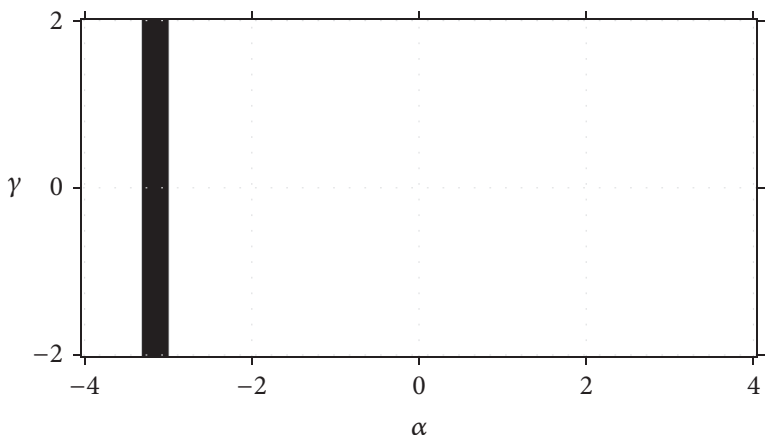

(a)

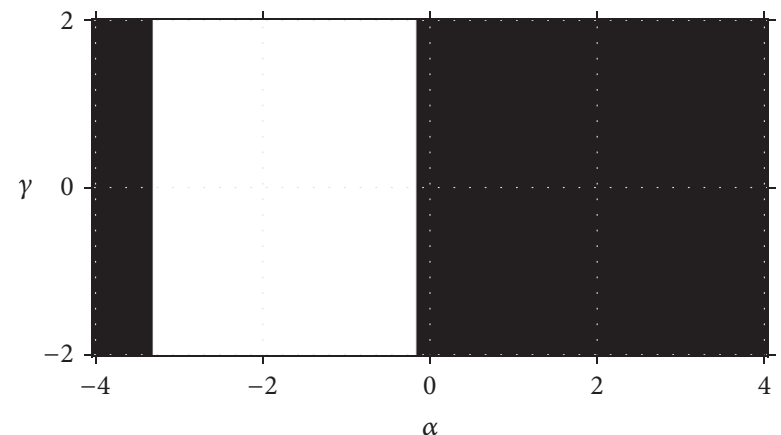

(b)

Figure 6: Stability plane of (a) $x_{1}^{F}$ and (b) $x_{2}^{F}$.

The polynomial has four real roots and four complex ones. No one of the critical points agrees with the roots of the function $x^{2}$, so every one is free. Figure 7 shows the parameters planes of each real critical point. When the critical point tends to $x_{1}^{*}$, $x_{2}^{*}$, or $x_{\infty}^{*}$, the points are plotted in red, green, or blue, respectively, while black is kept for convergence to any other point. Note in Figures 7(b), 7(c), and 7(d) there is a thin black region, where the critical points have periodical orbits, so they do not converge to any one of the fixed points.

For gathering the information of the parameters planes, the unified parameter plane is shown in Figure 8.

Finally, the dynamical behavior is analyzed with the dynamical line. The representation of the methods that converge to any of the roots is covered in Figure 9, while Figure 10 illustrates the methods that also converge to any other point or have a periodical orbit. Color map is held.

4.2.3. $f(x)=x^{2}+1$. Finally, the application of (32) on $f(x)=$ $x^{2}+1$ results in the fixed point operator $M=N_{11}(x) / D_{10}(x)$, where $N_{11}(x)$ and $D_{10}(x)$ are polynomials of degrees 11 and 10 , respectively.
The fixed points are the roots of $f(x)$ and the roots of an eighth-degree polynomial. Both groups of roots are complex, so they are left out of the purpose of the current study.

Regarding the critical points, an analogous comment can be made. The roots of $f(x)$ are critical points. The rest of the critical points are $x_{3,4}^{C}=-1 / \gamma \pm i$ and the roots of a polynomial of degree 12. No critical point is real, so the real dynamics can not be analyzed.

\section{Memory Dynamics}

Introducing memory in the iterative expression of a method can improve its order of convergence. Below some fundamentals about methods with memory are introduced [11, 17].

5.1. Basics on Iterative Methods with Memory. The standard form of an iterative method with memory of first order is

$$
x_{k+1}=\phi\left(x_{k}, x_{k-1}\right), \quad k \geq 1,
$$




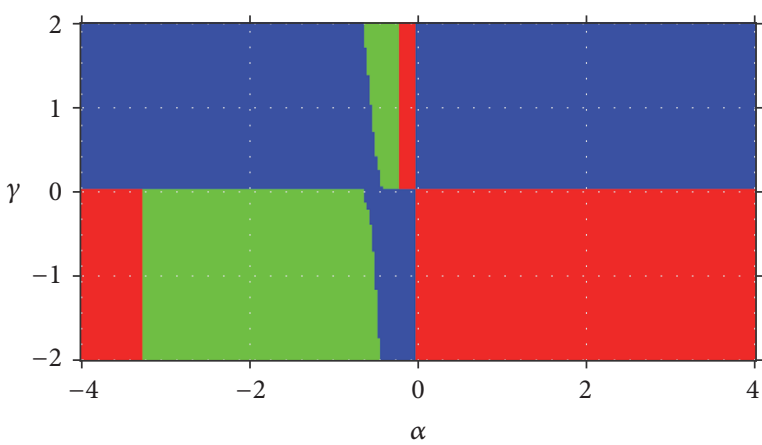

(a)

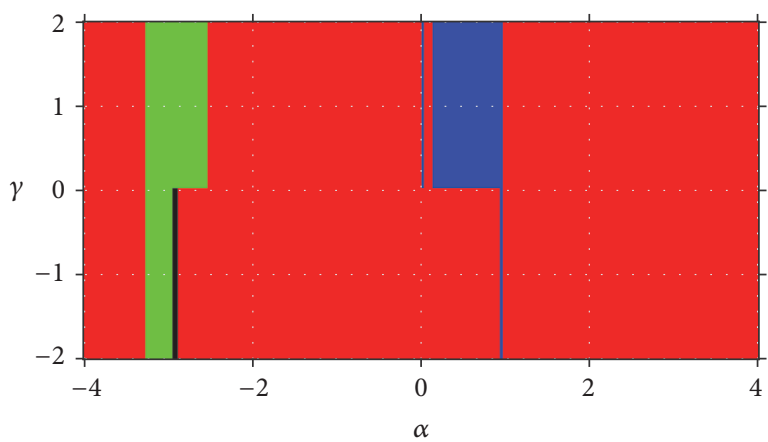

(c)

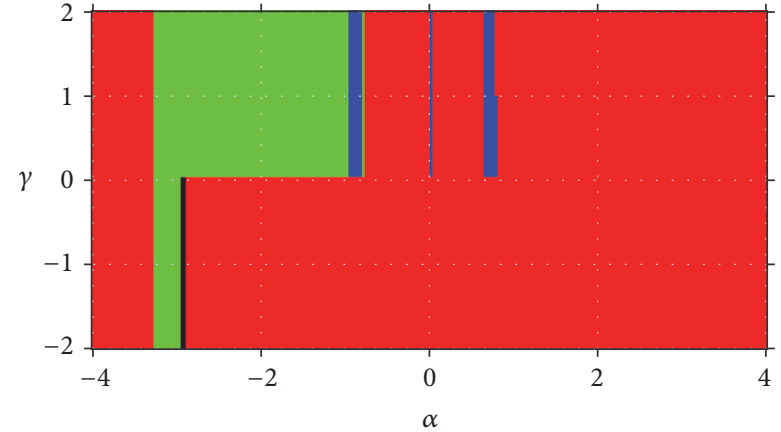

(b)

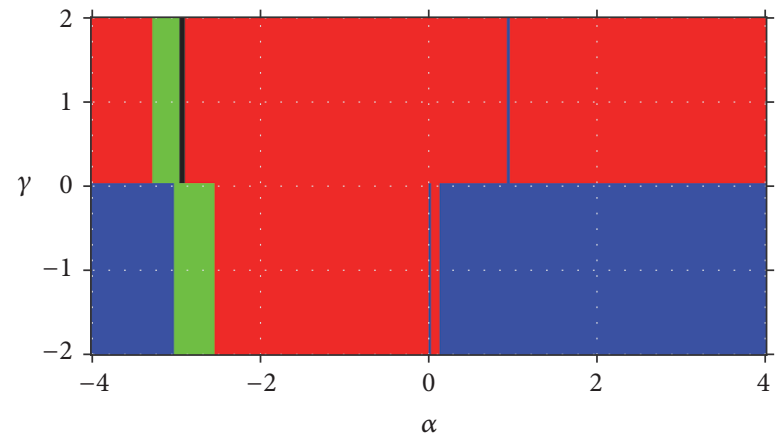

(d)

Figure 7: Parameter planes of (a) $x_{1}^{C}$, (b) $x_{2}^{C}$, (c) $x_{3}^{C}$, and (d) $x_{4}^{C}$ (red: $x_{1}^{*}$, green: $x_{2}^{*}$, blue: $x_{\infty}^{*}$, and black: others).

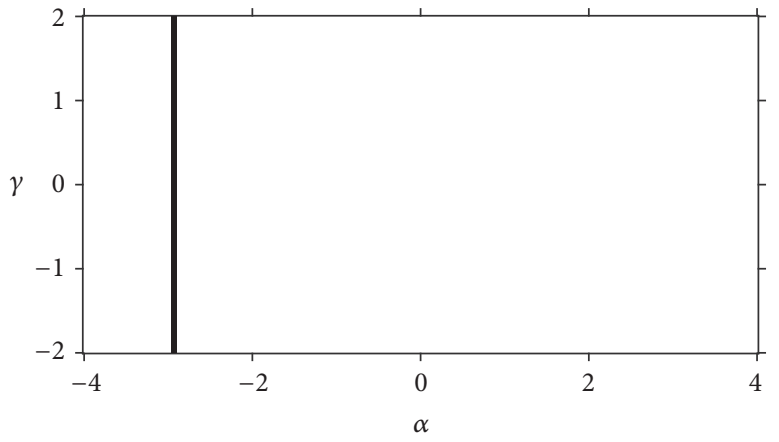

Figure 8: Unified parameter plane of $x_{1-4}^{C}$.

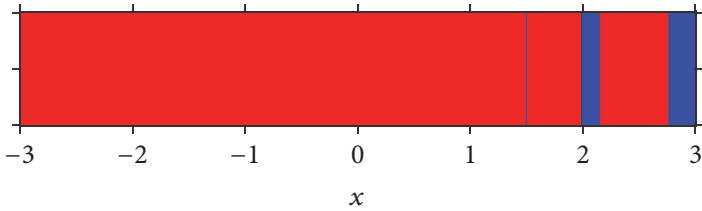

(a)

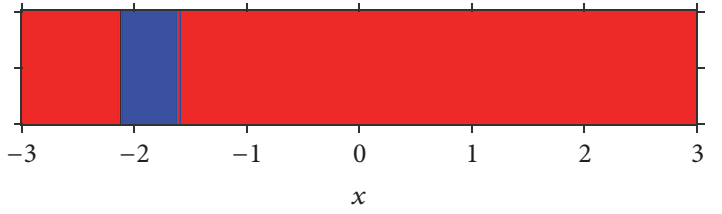

(c)

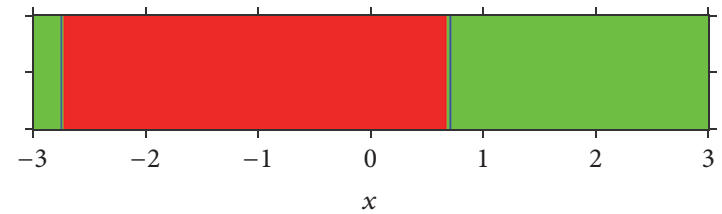

(b)

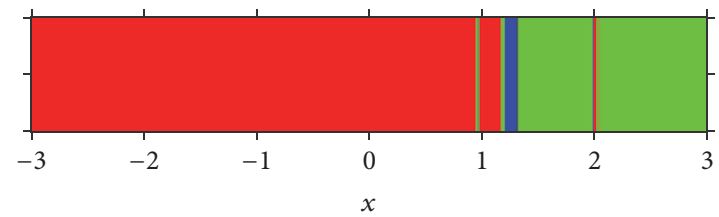

(d)

Figure 9: Dynamical lines of methods with (a) $(\alpha, \gamma)=(0.1124,-1)$, (b) $(\alpha, \gamma)=(-2,0.5),(\mathrm{c})(\alpha, \gamma)=(1,1)$, and $(\mathrm{d})(\alpha, \gamma)=(-0.23,-1.5)$ (red: $x_{1}^{*}$, green: $x_{2}^{*}$, blue: $x_{\infty}^{*}$, and black: others). 


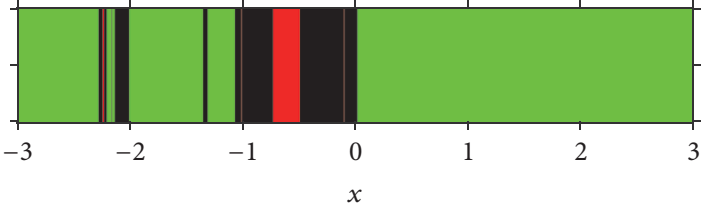

(a)

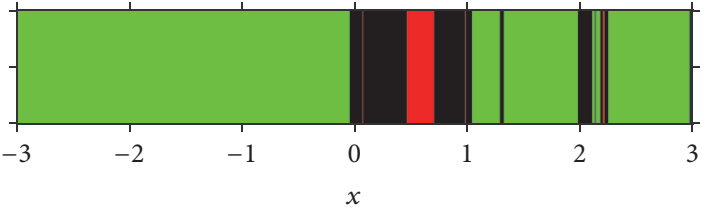

(b)

Figure 10: Dynamical lines of methods with $(\mathrm{a})(\alpha, \gamma)=(-2.876,1)$ and $(\mathrm{b})(\alpha, \gamma)=(-2.876,-1)$ (red: $x_{1}^{*}$, green: $x_{2}^{*}$, blue: $x_{\infty}^{*}$, and black: others).

where $x_{0}$ and $x_{1}$ are the initial estimations. Fixed points must satisfy two conditions: $x_{k+1}=x_{k}$ and $x_{k-1}=x_{k}$, so the fixed point function $\Phi$ can be defined by means of

$$
\begin{array}{r}
\Phi\left(x_{k-1}, x_{k}\right)=\left(x_{k}, x_{k+1}\right)=\left(x_{k}, \phi\left(x_{k-1}, x_{k}\right)\right), \\
k=1,2, \ldots .
\end{array}
$$

So, $\left(x_{k-1}, x_{k}\right)$ is a fixed point of $\Phi$ if $\Phi\left(x_{k-1}, x_{k}\right)=\left(x_{k-1}, x_{k}\right)$ and, consequently, $x_{k+1}=x_{k}$ and $x_{k}=x_{k-1}$. Therefore, the discrete dynamical system $\Phi: \mathbb{R}^{2} \rightarrow \mathbb{R}^{2}$, such that

$$
\Phi(\vec{x})=\Phi(z, x)=(x, \phi(z, x))
$$

where $\phi$ is the operator of the iterative scheme with memory, has been defined. Below some definitions related to iterative methods with memory are recalled.

The orbit of a point $\vec{x}_{0}$ is defined as the set $\{\vec{x}, \Phi(\vec{x})$, $\left.\Phi^{2}(\vec{x}), \ldots, \Phi^{n}(\vec{x}), \ldots\right\}$. A point $\vec{x}_{0}=(z, x)_{0}$ is a fixed point $\vec{x}_{0}^{F}=(z, x)_{0}^{F}$ of $\Phi$ if $z=x$ and $x=\phi(z, x)$. If a fixed point $\vec{x}^{F}$ of operator $\Phi$ is different from $(r, r)$, where $f(r)=0$, it is called strange fixed point. A point $\vec{x}_{T}$ is T-periodic if $\Phi^{T}\left(\vec{x}_{T}\right)=\vec{x}_{T}$ and $\Phi^{t}\left(\vec{x}_{T}\right) \neq \vec{x}_{T}$, for $t<T$.

The dynamical behavior of a point $\vec{x}$ is defined from its asymptotical behavior. For this purpose, Theorem 5.1 of [18] is recalled.

Theorem 5. Let $\Phi$ from $\mathbb{R}^{n}$ to $\mathbb{R}^{n}$ be $\mathscr{C}^{2}$. Assume $\vec{x}_{T}$ is $T$ periodic. Let $\lambda_{1}, \lambda_{2}, \ldots, \lambda_{n}$ be the eigenvalues of $\Phi^{\prime}\left(\vec{x}_{T}\right)$. Then

(1) if all the eigenvalues $\lambda_{j}$ have $\left|\lambda_{j}\right|<1$, then $\vec{x}_{T}$ is attracting;

(2) if one eigenvalue $\lambda_{j_{0}}$ has $\left|\lambda_{j_{0}}\right|>1$, then $\vec{x}_{T}$ is unstable, that is, repelling or saddle;

(3) if all the eigenvalues $\lambda_{j}$ have $\left|\lambda_{j}\right|>1$, then $\vec{x}_{T}$ is repelling.

In addition,

(1) if all the eigenvalues $\lambda_{j}$ have $\left|\lambda_{j}\right| \neq 1$, the fixed point is hyperbolic;

(2) if there exist an eigenvalue $\lambda_{i}:\left|\lambda_{i}\right|<1$ and another eigenvalue $\lambda_{j}:\left|\lambda_{j}\right|>1$, then the hyperbolic point is recalled as saddle point.
A critical point $\vec{x}^{C}$ satisfies $\operatorname{det}\left(\Phi^{\prime}\left(\vec{x}^{C}\right)\right)=0$. The basin of attraction of a fixed attracting point, $\mathscr{A}\left(\vec{x}^{*}\right)$, is defined as the set of preimages of any order such that

$$
\mathscr{A}\left(\vec{x}^{*}\right)=\left\{\vec{x}_{0} \in \mathbb{R}^{n}: \Phi^{m}\left(\vec{x}_{0}\right) \longrightarrow \vec{x}^{*}, m \longrightarrow \infty\right\} .
$$

The drawing tool to represent the basins of attraction is the dynamical plane $[16,19]$. This tool is based on the application to complex dynamics, where horizontal and vertical axes stand for the real and imaginary parts of a complex value, respectively. For real dynamics with memory, the horizontal axis is devoted to the current iteration $x_{k}$ while the vertical one represents the last iteration $x_{k-1}$.

5.2. Stability of Family $M M 1(\alpha)$. As we have seen in Section 3 , the memory is introduced in the iterative expression by replacing parameter $\gamma$ by $-1 / \overline{f^{\prime}}\left(x^{*}\right)$, where $\overline{f^{\prime}}\left(x^{*}\right)$ denotes any approximation of $f^{\prime}\left(x^{*}\right)$. The first family of iterative methods with memory designed in Section 3 is expressed as

$$
\begin{aligned}
w_{k} & =x_{k}-\frac{f\left(x_{k}\right)}{f\left[x_{k}, x_{k-1}\right]}, \\
y_{k} & =x_{k}-\frac{f\left(x_{k}\right)}{f\left[x_{k}, w_{k}\right]}, \\
x_{k+1} & =y_{k}-\frac{f\left(x_{k}\right)+\alpha f\left(y_{k}\right)}{f\left(x_{k}\right)+(\alpha-1) f\left(y_{k}\right)} \frac{f\left(y_{k}\right)}{f\left[y_{k}, w_{k}\right]},
\end{aligned}
$$

where $\alpha$ is a free parameter. We are going to analyze the dynamical behavior of this class on the quadratic polynomials $x^{2}-1, x^{2}$, and $x^{2}+1$.

5.2.1. $f(x)=x^{2}-1$. When (39) is applied over $f(x)=x^{2}-1$, the fixed point operator is

$$
\begin{aligned}
& M_{\alpha}(z, x)=\left(-\left(x^{2}-1\right)^{2}\left(z^{2}-1\right)(x+z)\right. \\
& \quad \cdot\left(a\left(x^{2}-1\right)\left(z^{2}-1\right)+\left(x^{2}+2 x z+1\right)^{2}\right) /\left(2 x^{3} z\right. \\
& \left.+3 x^{2}\left(z^{2}+1\right)+6 x z+z^{2}+1\right) \\
& \quad \cdot\left(a\left(x^{2}-1\right)\left(z^{2}-1\right)\right. \\
& \left.+(x+z)\left(x\left(x^{2}+3 x z+3\right)+z\right)\right)+x^{2} z+2 x \\
& +z)\left(x^{2}+2 x z+1\right)^{-1} .
\end{aligned}
$$




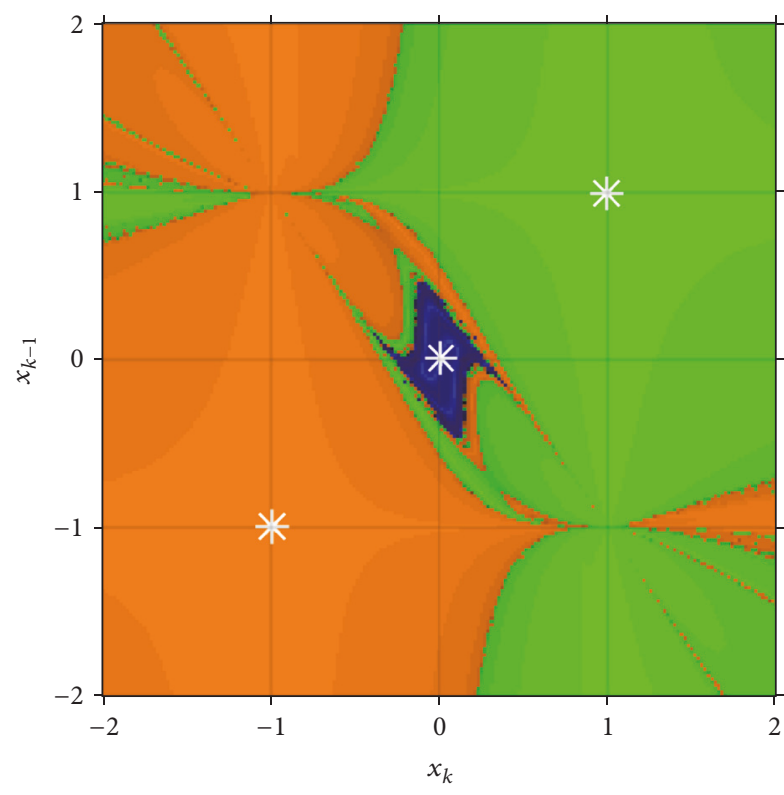

(a) $\alpha=-0.47$, and $z_{2}^{F}$ is attracting

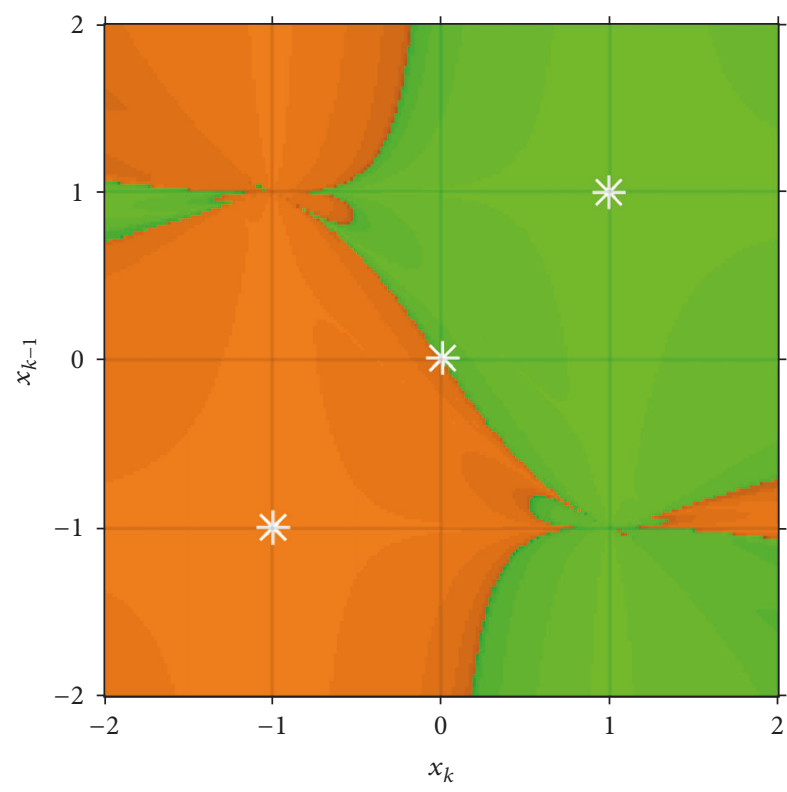

(b) $\alpha=0.5$, and $z_{2}^{F}$ is repelling

FIGURE 11: Dynamical planes of method $M_{\alpha}(z, x)$ for $f(x)=x^{2}-1$.

The fixed points of the operator are $(z, x)_{1,2,3}^{F}=$ $\{(-1,-1),(0,0),(1,1)\}$. The fixed points $(z, x)_{1}^{F}=(z, x)_{1}^{*}=$ $(-1,-1)$ and $(z, x)_{3}^{F}=(z, x)_{3}^{*}=(1,1)$ are superattracting, while the behavior of $(z, x)_{2}^{F}$ depends on the parameter $\alpha$. In this way, $(z, x)_{2}^{F}=(z, x)_{2}^{*}$ is attracting just in case $-1 / 2<\alpha \leq$ $-(1 / 17)(5+2 \sqrt{2})$.

Figure 11 represents the dynamical plane of the method when $(z, x)_{2}^{F}$ is attracting (a) and when it is not (b). White stars plot the attracting points. Orange points represent the set of initial points that converge to $(z, x)_{1}^{*}=(-1,-1)$, while green points are the $(z, x)_{3}^{*}=(1,1)$ corresponding ones. Blue is devoted to initial estimations that finally converge to $(z, x)_{2}^{*}=(0,0)$. The initial points are a mesh of $501 \times 501$ points of values of $x$ and $z$ from -2 to 2 . Every initial point has converged to one of the attracting fixed points in less than 100 iterations. Further information about the representation can be found in [16].

5.2.2. $f(x)=x^{2}$. The application of (39) over $f(x)=x^{2}$ results in the following fixed point operator:

$$
\begin{aligned}
& M_{\alpha}(z, x) \\
& \quad=\frac{x z\left(x^{3}+6 x^{2} z+\alpha x z^{2}+10 x z^{2}+2 \alpha z^{3}+5 z^{3}\right)}{(x+2 z)(2 x+3 z)\left(x^{2}+4 x z+\alpha z^{2}+3 z^{2}\right)} .
\end{aligned}
$$

The fixed points are $(z, x)_{1}^{F}=(0,0)$ and $(z, x)_{\infty}^{F}=\infty$. Some singularities can be found when the denominator of (41) is zero, for $z=-x / 2, z=-2 x / 3$, and $\alpha=-8$. This behavior is illustrated in Figure 12, where two dynamical planes are represented for $\alpha \neq-8$ (a) and $\alpha=-8$ (b).
5.2.3. $f(x)=x^{2}+1$. Finally, we apply (39) on $f(x)=$ $x^{2}+1$. The fixed point operator is a rational function whose numerator has degree 9 and its denominator has degree 8 . Note the degrees are the higher sum of the powers of $x$ and $z$. Let $r_{1-8}$ be the roots of $p(x)=x^{8}(6 \alpha+49)+x^{6}(4 \alpha-108)+$ $x^{4}(86-8 \alpha)+x^{2}(-4 \alpha-12)+2 \alpha+1$. Depending on the region of $\alpha$, the amount of fixed points changes. Table 1 summarizes the fixed points depending on the value of $\alpha$.

Every fixed point whose value is known is repelling, except the cases collected in Table 2. Related to Theorem 5, only the point $(0,0)$ is an attracting fixed point for $\alpha=$ -0.381966 ; the rest of the points are nonhyperbolic.

The dynamical planes of the cases in Table 2 are represented in Figures 13(a)-13(d). In this case, white stars represent the fixed points. Only for the particular case of $\alpha=$ -0.381966 is there an attracting fixed point that attracts a few points of the memory plane, as can be observed in Figure 13.

Since the usual behavior is the nonconvergence to any of the roots, the orbit of different initial points have been obtained and represented in Figures 13(e)-13(h).

For $\alpha \in\{-0.5,-0.324219,4.73341\}$, whose corresponding Figures are (e), (g), and (h), the orbit of the analyzed points falls into a periodical orbit, as the magnification in their insets shows.

\section{Conclusions}

We have designed a biparametric family of fourth-order optimal schemes for solving nonlinear equations. Its error equation allows us to introduce memory increasing the order of convergence until six, without need of more functional evaluations. We have considered in depth the dynamical study of 


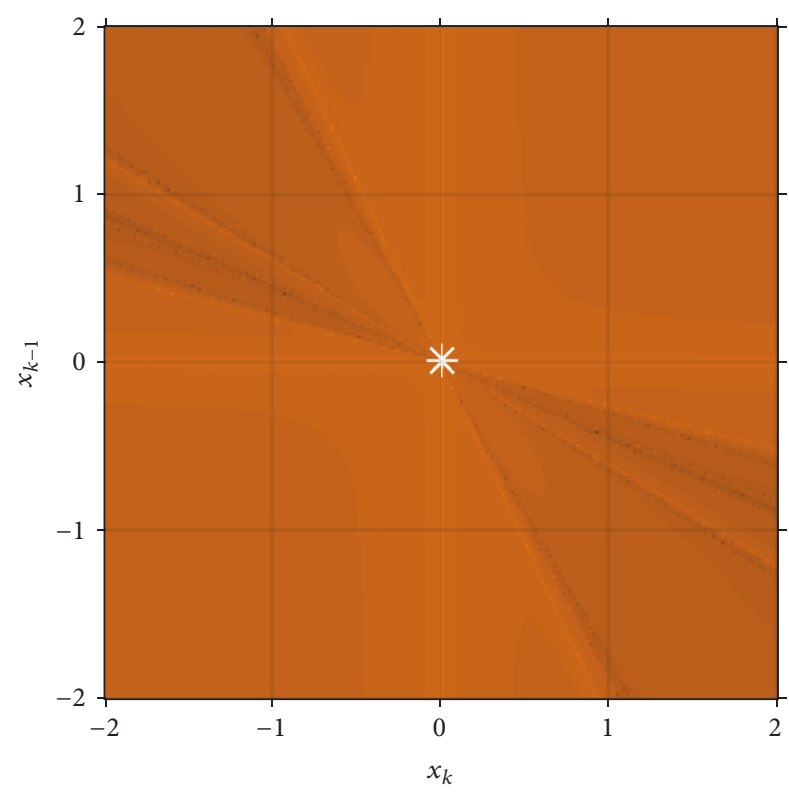

(a) $\alpha=-1$

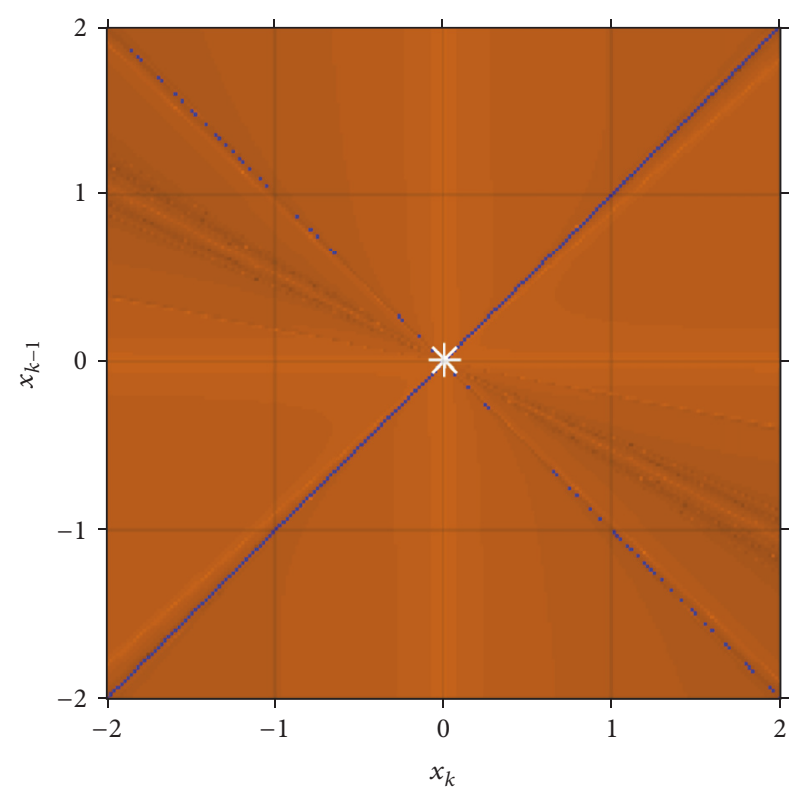

(b) $\alpha=-8$

FIGURE 12: Dynamical planes of method $M_{\alpha}(z, x)$ for $f(x)=x^{2}$.

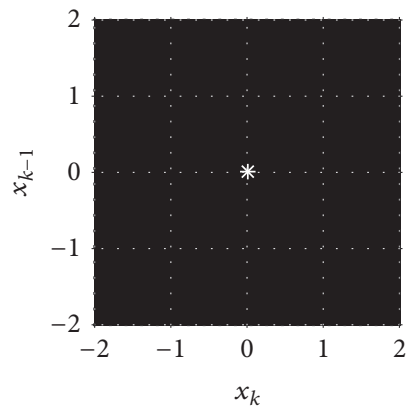

(a) $\alpha=-0.5$
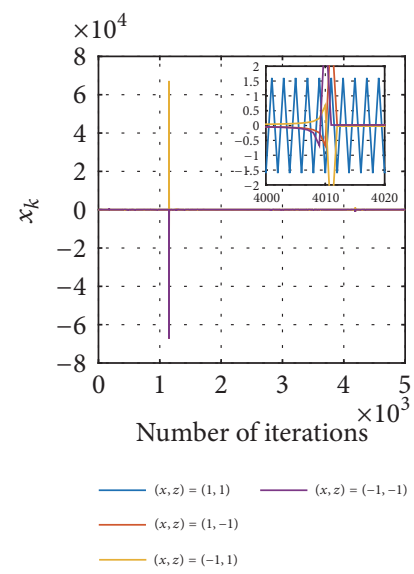

(e) $\alpha=-0.5$

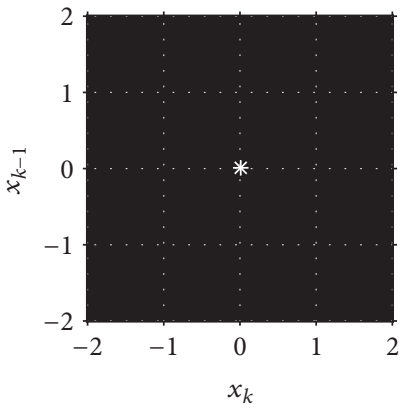

(b) $\alpha=-0.381966$
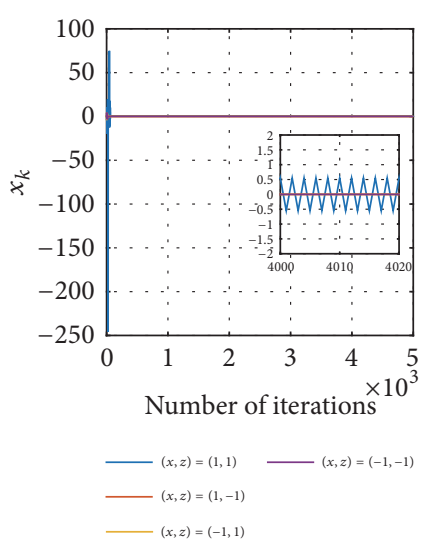

(f) $\alpha=-0.381966$

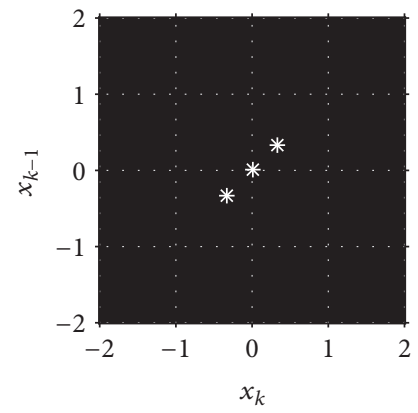

(c) $\alpha=-0.324219$

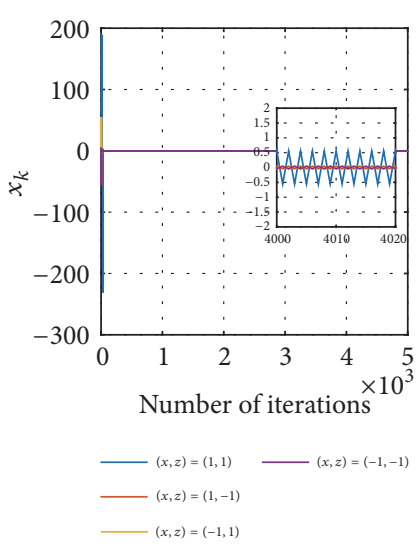

(g) $\alpha=-0.324219$

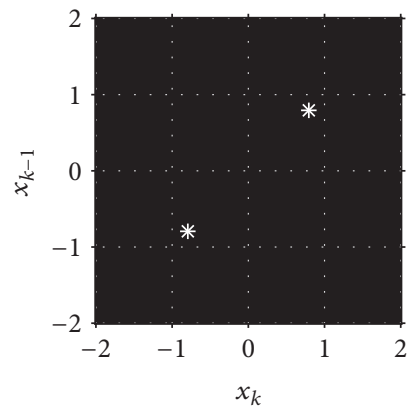

(d) $\alpha=4.73341$

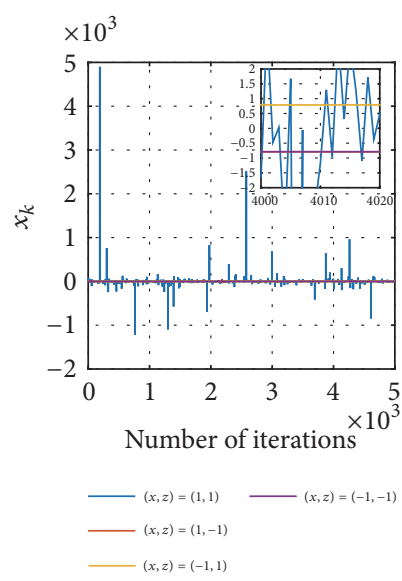

(h) $\alpha=4.73341$

FIGURE 13: ((a)-(d)) Dynamical planes of method $M_{\alpha}(z, x)$ for $f(x)=x^{2}+1$. ((e)-(h)) Orbit of points $x_{k}$ for 5000 iterations; inset: orbit at iterations 4000 to 4020 . 
TABLE 1: Fixed points depending on $\alpha$.

\begin{tabular}{lr}
\hline$\alpha$ & Fixed points \\
\hline$<-8.1667$ & $\left(r_{1}, r_{1}\right),\left(r_{2}, r_{2}\right),\left(r_{3}, r_{3}\right),\left(r_{4}, r_{4}\right),(0,0)$ \\
$\epsilon(-8.1667,-2.61803)$ & $\left(r_{1}, r_{1}\right),\left(r_{2}, r_{2}\right),\left(r_{3}, r_{3}\right),\left(r_{4}, r_{4}\right),\left(r_{5}, r_{5}\right),\left(r_{6}, r_{6}\right),(0,0)$ \\
$=-2.61803$ & $( \pm 1.26518, \pm 1.26518),( \pm 0.488916, \pm 0.488916),(0,0)$ \\
\hline$\epsilon(-2.61803,-2.57392)$ & $\left(r_{1}, r_{1}\right),\left(r_{2}, r_{2}\right),\left(r_{3}, r_{3}\right),\left(r_{4}, r_{4}\right),\left(r_{5}, r_{5}\right),\left(r_{6}, r_{6}\right),(0,0)$ \\
$=-2.57392$ & $( \pm 1.31541, \pm 1.31541),( \pm 0.487946, \pm 0.487946),(0,0)$ \\
$\epsilon(-2.57392,-0.5)$ & $\left(r_{1}, r_{1}\right),\left(r_{2}, r_{2}\right),(0,0)$ \\
\hline$=-0.5$ & $( \pm 0.361815, \pm 0.361815),(0,0)$ \\
$\in(-0.5,-0.381966)$ & $\left(r_{1}, r_{1}\right),\left(r_{2}, r_{2}\right),\left(r_{3}, r_{3}\right),\left(r_{4}, r_{4}\right),(0,0)$ \\
$=-0.381966$ & $( \pm 0.172745, \pm 0.172745),(0,0)$ \\
\hline$\epsilon(-0.381966,-0.324219)$ & $\left(r_{1}, r_{1}\right),\left(r_{2}, r_{2}\right),\left(r_{3}, r_{3}\right),\left(r_{4}, r_{4}\right),(0,0)$ \\
$=-0.324219$ & $( \pm 0.262345, \pm 0.262345),(0,0)$ \\
$\epsilon(-0.324219,0)$ & $(0,0)$ \\
\hline$\epsilon(0,4.73341)$ & $(0,0)$ \\
$=4.73341$ & $( \pm 0.788301, \pm 0.788301),(0,0)$ \\
$>4.73341$ & $\left(r_{1}, r_{1}\right),\left(r_{2}, r_{2}\right),\left(r_{3}, r_{3}\right),\left(r_{4}, r_{4}\right),(0,0)$ \\
\hline
\end{tabular}

TABLE 2: Fixed points without repelling behavior.

\begin{tabular}{lcc}
\hline$\alpha$ & Fixed points & Eigenvalues \\
\hline-0.5 & $(0,0)$ & $(0,1)$ \\
-0.381966 & $(0,0)$ & $0.190983+0.7626 i(-1,1),\left|\lambda_{1,2}\right|=0.786151$ \\
-0.324219 & $( \pm 0.262345, \pm 0.262345)$ & $(0.438608,1)$ \\
-0.324219 & $(0,0)$ & $-0.0421652+1.04046 i(-1,1),\left|\lambda_{1,2}\right|=1.04131$ \\
4.73341 & $( \pm 0.788301, \pm 0.788301)$ & $(-0.613745,1)$ \\
\hline
\end{tabular}

this family. Several tools related to real and memory dynamics have been applied, supplying a very important visual information about the different behaviors. Moreover, the dynamical line and the unified parameter plane have been introduced for the first time to the best of our knowledge.

The real dynamics of the biparametric family has revealed its good performance in terms of stability. For polynomials with complex roots, the iterative family does not fail, while for the rest of polynomials there are wide regions for both parameters where the stability of the family is guaranteed.

The memory dynamics have improved the order of convergence of the original method. For polynomials with real roots, the iterative family of methods has a broad stable behavior. When the family is applied over polynomials with complex roots, many fixed points with repelling behavior appear, and some periodical orbits can be found.

\section{Conflicts of Interest}

The authors declare that there are no conflicts of interest regarding the publication of this paper.

\section{Acknowledgments}

This research was partially supported by Ministerio de Economía y Competitividad under Grants MTM2014-52016C2-2-P, Generalitat Valenciana PROMETEO/2016/089, and FONDOCYT 2014-1C1-088 República Dominicana.

\section{References}

[1] F. Chicharro, A. Cordero, J. . Gutiérrez, and J. R. Torregrosa, "Complex dynamics of derivative-free methods for nonlinear equations," Applied Mathematics and Computation, vol. 219, no. 12, pp. 7023-7035, 2013.

[2] F. I. Chicharro, A. Cordero, and J. R. Torregrosa, "Dynamics and fractal dimension of Steffensen-type methods," Algorithms, vol. 8, no. 2, pp. 271-279, 2015.

[3] J. L. Hueso, E. Martínez, and C. Teruel, "Derivative free iterative methods for nonlinear systems," Applied Mathematics and Computation, vol. 259, pp. 955-966, 2015.

[4] Á. A. Magreñán, "A new tool to study real dynamics: the convergence plane," Applied Mathematics and Computation, vol. 248, pp. 215-224, 2014.

[5] Á. A. Magreñán, A. Cordero, J. M. Gutiérrez, and J. R. Torregrosa, "Real qualitative behavior of a fourth-order family of iterative methods by using the convergence plane," Mathematics and Computers in Simulation, vol. 105, pp. 49-61, 2014.

[6] H. T. Kung and J. F. Traub, "Optimal order of one-point and multipoint iteration," Journal of the ACM, vol. 21, pp. 643-651, 1974.

[7] A. M. Ostrowski, Solution of Equations and Systems of Equations, Pure and Applied Mathematics, Vol. IX. Academic Press, New York, NY, USA, 1960.

[8] J. F. Traub, Iterative Methods for the Solution of Equations, Prentice-Hall, New York, NY, USA, 1964.

[9] M. S. Petković, B. Neta, L. D. Petković, and J. Džunić, Multipoint Methods for Solving Nonlinear Equations, Academic Press, Amsterdam, The Netherlands, 2013. 
[10] M. S. Petkovic', B. Neta, L. D. Petkovic', and J. Džunic', "Multipoint methods for solving nonlinear equations: a survey," Applied Mathematics and Computation, vol. 226, pp. 635-660, 2014.

[11] B. Campos, A. Cordero, J. R. Torregrosa, and P. Vindel, "A multidimensional dynamical approach to iterative methods with memory," Applied Mathematics and Computation, vol. 271, pp. 701-715, 2015.

[12] J. M. Ortega and W. C. Rheinboldt, Solutions of equations and systems of equations, Academic Press, New York, NY, USA, 1960.

[13] P. Blanchard, "Complex analytic dynamics on the Riemann sphere," Bulletin of the American Mathematical Society, vol. 11, no. 1, pp. 85-141, 1984.

[14] R. L. Devaney, An Introduction to Chaotic Dynamical Systems, Addison-Wesley, Redwood, Calif, USA, 1st edition, 1989.

[15] J. M. Gutiérrez, S. Plaza, and N. Romero, "Dynamics of a fifth-order iterative method," International Journal of Computer Mathematics, vol. 89, no. 6, pp. 822-835, 2012.

[16] F. Chicharro, A. Cordero, and J. R. Torregrosa, "Drawing dynamical and parameter planes of iterative families and methods," The Scientific World Journal, vol. 2013, Article ID 780153, 11 pages, 2013.

[17] B. Campos, A. Cordero, J. R. Torregrosa, and P. Vindel, "Stability of king's family of iterative methods with memory," Journal of Computational and Applied Mathematics, vol. 318, pp. 504-514, 2017.

[18] R. C. Robinson, An Introduction to Dynamical Systems-Continuous and Discrete, vol. 19 of Pure and Applied Undergraduate Texts, American Mathematical Society, Providence, Second edition, 2012.

[19] J. L. Varona, "Graphic and numerical comparison between iterative methods," The Mathematical Intelligencer, vol. 24, no. 1, pp. 37-46, 2002. 


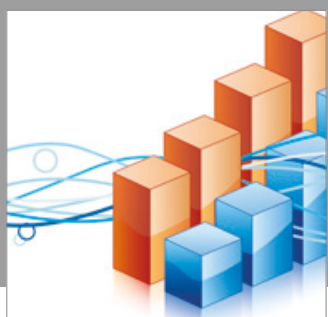

Advances in

Operations Research

vatersals

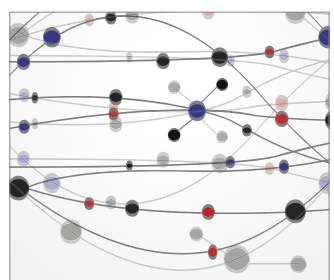

\section{The Scientific} World Journal
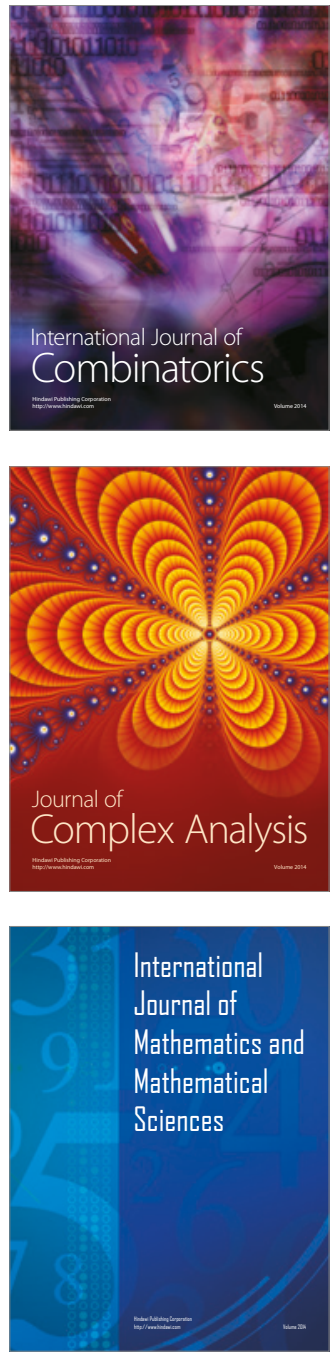
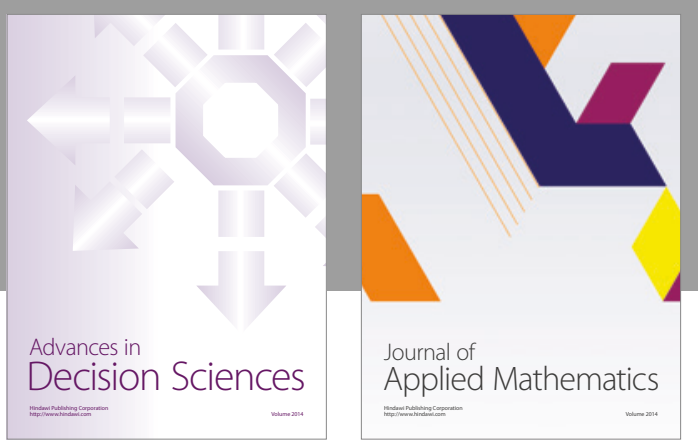

Algebra

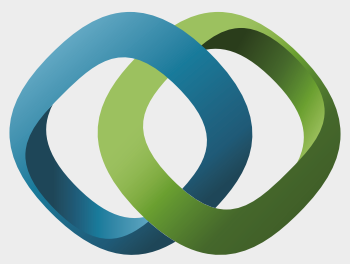

\section{Hindawi}

Submit your manuscripts at

https://www.hindawi.com
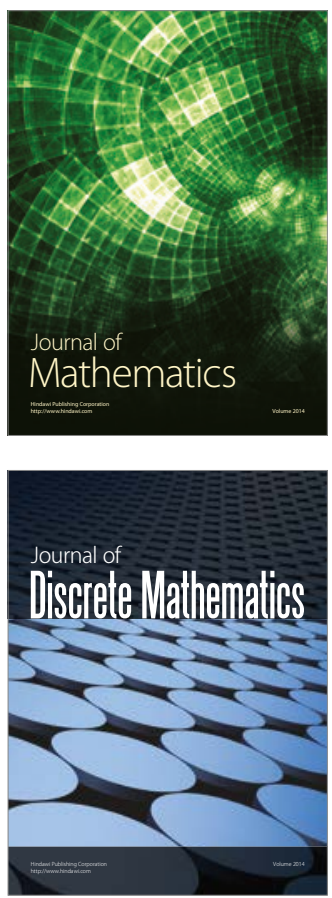

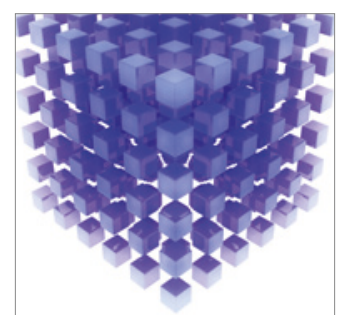

Mathematical Problems in Engineering
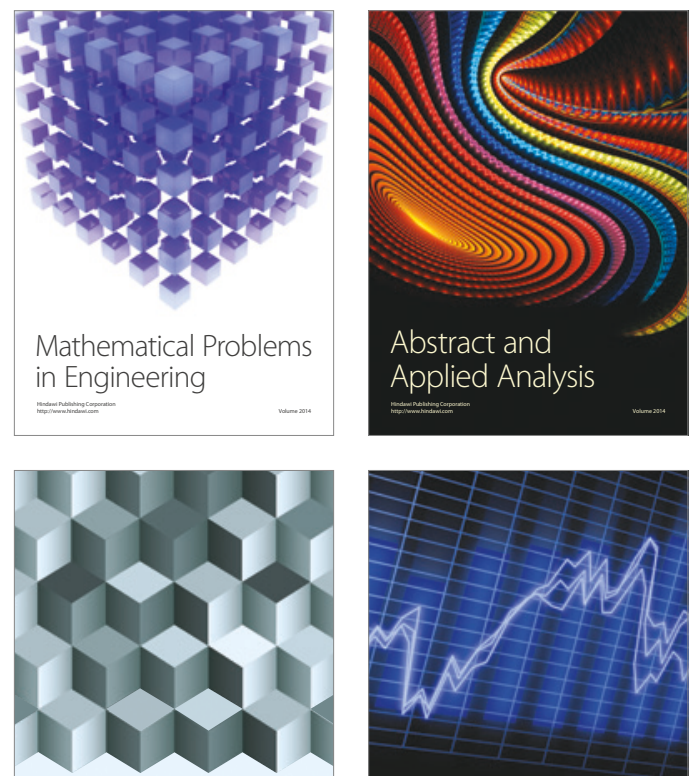

Journal of

Function Spaces

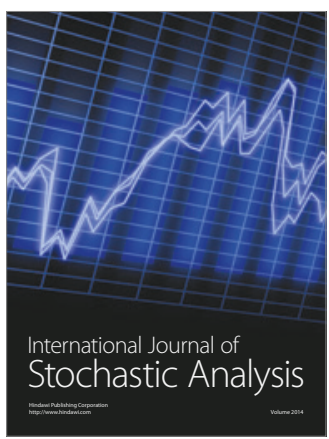

Probability and Statistics
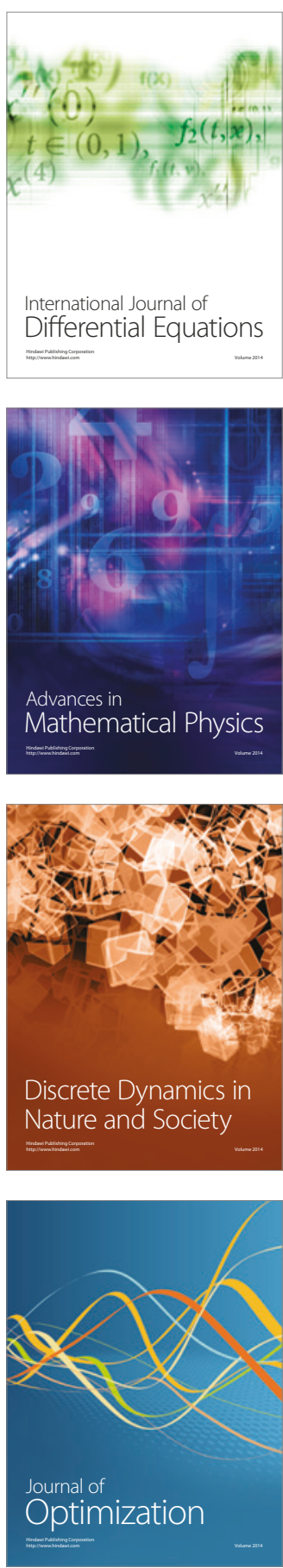